Elsevier Editorial System(tm) for Finite Elements in Analysis and Design

Manuscript Draft

Manuscript Number: L1898

Title: Optimization of Stability Constrained Geometrically Nonlinear Shallow Trusses Using an Arc Length Sparse Method with a Strain Energy Density Approach

Article Type: Original Research Paper

Section/Category:

Keywords: Optimization, Stability Constrained, Geometrically Nonlinear, Trusses, Arc Length, Sparse, Strain Energy Density

Corresponding Author: Senior Structural Engineer Glenn Andrew Hrinda, MS Civil Engineering

Corresponding Author's Institution: NASA Langley Research Center

First Author: Glenn A Hrinda, M.S. Civil Engineering

Order of Authors: Glenn A Hrinda, M.S. Civil Engineering; Duc T Nguyen, Ph.D. Civil Engineering

Manuscript Region of Origin:

Abstract: A technique for the optimization of stability constrained geometrically nonlinear shallow trusses with snap through behavior is demonstrated using the arc length method and a strain energy density approach within a discrete finite element formulation. The optimization method uses an iterative scheme that evaluates the design variables' performance and then updates them according to a recursive formula controlled by the arc length method. A minimum weight design is achieved when a uniform nonlinear strain energy density is found in all members. This minimal condition places the design load just below the critical limit load causing snap through of the structure. The optimization scheme is programmed into a nonlinear finite element algorithm to find the large strain energy at critical limit loads. Examples of highly nonlinear trusses found in literature are presented to verify the method. 



\title{
Optimization of Stability Constrained Geometrically Nonlinear Shallow Trusses Using an Arc Length Sparse Method with a Strain Energy Density Approach
}

\begin{abstract}
A technique for the optimization of stability constrained geometrically nonlinear shallow trusses with snap through behavior is demonstrated using the arc length method and a strain energy density approach within a discrete finite element formulation. The optimization method uses an iterative scheme that evaluates the design variables' performance and then updates them according to a recursive formula controlled by the arc length method. A minimum weight design is achieved when a uniform nonlinear strain energy density is found in all members. This minimal condition places the design load just below the critical limit load causing snap through of the structure. The optimization scheme is programmed into a nonlinear finite element algorithm to find the large strain energy at critical limit loads. Examples of highly nonlinear trusses found in literature are presented to verify the method.
\end{abstract}




\title{
Optimization of Stability Constrained Geometrically Nonlinear Shallow Trusses Using an Arc Length Sparse Method with a Strain Energy Density Approach
}

\begin{abstract}
A technique for the optimization of stability constrained geometrically nonlinear shallow trusses with snap through behavior is demonstrated using the arc length method and a strain energy density approach within a discrete finite element formulation. The optimization method uses an iterative scheme that evaluates the design variables' performance and then updates them according to a recursive formula controlled by the arc length method. A minimum weight design is achieved when a uniform nonlinear strain energy density is found in all members. This minimal condition places the design load just below the critical limit load causing snap through of the structure. The optimization scheme is programmed into a nonlinear finite element algorithm to find the large strain energy at critical limit loads. Examples of highly nonlinear trusses found in literature are presented to verify the method.
\end{abstract}

\section{Introduction}

Modern design of light weight truss structures have been achieved through analysis techniques developed to investigate nonlinear instabilities. Appling loads to a truss system that produce non-proportional displacements is a nonlinear behavior. Increases in loading will make the structure unstable or buckle. In [13], these systems are called highly flexible structures (HFS) that are designed for large elastic displacements and without any plastic deformation. These HFS may either show bifurcation or limit point instability. Bifurcation instability occurs when the structure has a sudden geometric change and is characterized by an abrupt equilibrium path change along the load path. Strain energy from a compressive force is converted to bending strain energy without a large force increase. This can best be visualized as the typical Euler buckling of a slender column. The second type of nonlinear structural response is limit point instability. This behavior is displayed by a gradually changing equilibrium path without any sudden changes. Increasing the load beyond the limit point will cause a release of strain energy as the stiffness becomes negative.

Light weight truss structures are often highly flexible with unpredictable nonlinear responses and may become unstable before a bifurcation point is reached. An understanding of elastic stability behavior in nonlinear structures can be gained by graphically representing the load and displacement called the equilibrium path of the structure.

Methods that overcome the challenges of accurately identifying nonlinear equilibrium paths have been improving and also have developed to include optimization at limit points. A growing number of methods have been attempted to optimize a structure at a limit load with varying success. The challenge is to design a flexible structure undergoing large displacements that can still remain functional without a loss of stability. 
Large aerospace truss systems have been proposed over the years to support future on orbit space technologies. An example, that started in the 1960's, has been the repeated support for deploying space based solar power from various research organizations. The United States Department of Defense is currently studying how to demonstrate a deployed solar power constellation to supply power to remote bases or on the battlefield. Also during the 1960's, Rockwell International began studying large space truss structures for supporting microwave antennas and mirrors [5]. Another example of space trusses is today's International Space Station that makes use of deployable trusses to support its solar array panels. All of these space based concepts rely on the truss system to support their mission goals because a truss is the easiest to assemble in micro gravity and offers a minimum weight design. The lighter and more efficient these structural systems become with very slender members, the more likely they will have nonlinear stability problems. The analysis challenge is to find effective techniques that go beyond finding Euler type bifurcation points and find the optimal truss to carry applied limit loads without becoming unstable and releasing stored strain energy.

Weight optimization of slender truss members with stability constraints has been investigated by other researchers using various analytical techniques. Prager [14] discusses a general method of optimality subject to a single design constraint set of displacement, critical buckling load or natural frequency. Prager and Taylor [15] investigated columns under linear buckling constraints. Other investigators followed with schemes to optimize columns with multiple eigenvalues and design sensitivities with constraints on buckling. Some of the earliest finite element optimization investigations started in the 1970's with linear buckling that ignored any large deformations. Khot, Venkayya and Berke in [9] presented an optimality criterion for minimizing weight to structures with stability requirements using a linear eigenvalue buckling solution within a finite element analysis. Several other published works have studied nonlinear buckling of shallow trusses. Kamat and Ruangsilasingha [6] addressed the problem of maximizing the critical load of shallow three dimensional trusses. They developed sensitivity derivatives of the critical load with respect to design variables. Many case studies used in previous published works were taken from Crisfield [3, 4]. The case examples he used challenged many nonlinear solvers to correctly follow a nonlinear snap through load path. Crisfield used these problems to test nonlinear behavior in truss structures and demonstrate the importance of identifying the critical limit load along the equilibrium path. In [8] Kamat, Khot and Venkayya offer exact closed form solutions to simple shallow truss problems optimized for weight. They make use of the minimization of total potential energy that maximizes the load carrying capability without instability. Optimizations of geometrically nonlinear trusses similar to those used by Crisfield were attempted later by Khot and Kamat in [7]. These examples highlighted the challenges many analysis techniques have in following a nonlinear response path up to a critical limit load. The example problems also fully exposed the short comings of modern finite element programs through their lack of any optimization scheme for stability constrained problems. Sedaghati [16] developed an optimization technique using the Group Theoretic Approach. The method had stability constraints that used a strain energy density recurrence relationship for updating design variables. The technique centers around obtaining a uniform strain energy density in all members to establish a minimum weight design. They further made use of shallow type flexible truss structures to test and verify their methods. 
The method used in this paper achieves an optimal design using the principal of strain energy density and parameters in the arc length method to trace the nonlinear equilibrium path up to an instability point. A stable structural system exists when deformations are increasing as the applied load is increased and unstable when deformations are still increasing but the loads are decreasing.

An approach combining the arc length method with a design variable update scheme that finds a uniform nonlinear strain energy density in all members is programmed into a finite element algorithm. The optimization method uses an iterative scheme that evaluates the design variables' performance and then updates them according to a recursive formula controlled by the arc length method. Examples of highly nonlinear trusses found in literature are presented to verify the method. Only conservative systems that adhere to the theorem of minimum potential energy are considered. These are stable systems subjected to quasi-static proportional loads where the equations of equilibrium make the potential energy stationary under small displacements [18].

\section{Review of Arc Length Formulas}

Various versions of the arc length method have been presented by many researchers $[2-4,13$, $17,19]$ and incorporated into the finite element analysis procedures. For convenience to the readers and to facilitate the discussion in subsequent sections, the Riks-Wempner arc length method is reviewed in the following paragraphs. The Riks-Wempner method is considered a partial arc length method because it relies on a normal to the tangent rather than the circular path to search for an equilibrium point [13]. The method traces the nonlinear equilibrium path using an iterative process that begins with computing initial displacements, $\Delta q_{0}$ due to a user defined load increment $\Delta \lambda_{0}$, as shown in figure 1. The linear stiffness, $K_{T_{0}}$, is used to start the process and is replaced with the tangent stiffness, $K_{T_{i}}$, in further iterations.

The initial displacements $\Delta q_{0}$ are found using

$$
\frac{\Delta \lambda_{0}}{\Delta q_{0}}=\frac{\lambda}{\Delta q_{t o t}}
$$

where $\lambda=1$ and $\Delta q_{t o t}$ is found from the expression

$K_{T_{0}} \Delta q_{\text {tot }}=\lambda Q$

with $Q$ being the total applied loading. The notations given here for the displacements and loads are vector quantities with the stiffness notation understood as a matrix. Substituting into (2.1) gives

$\frac{\Delta \lambda_{0}}{\Delta q_{0}}=\frac{\lambda=1}{\left(\Delta q_{\text {tot }}\right)_{0}}$

that is solved for the initial displacement of

$\Delta q_{0}=\Delta \lambda_{0}\left(\Delta q_{t o t}\right)_{0}$ 


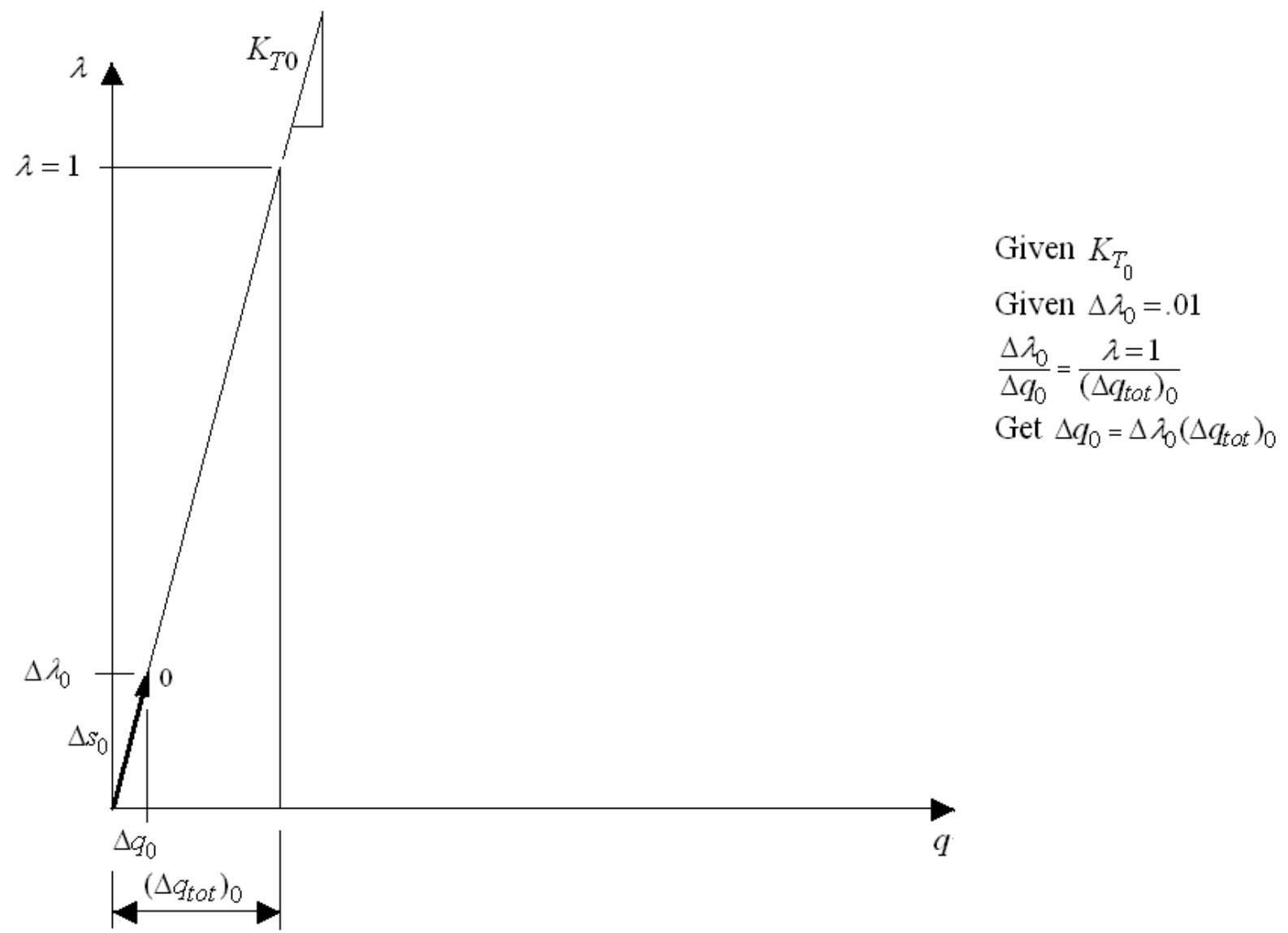

Figure 1. Starting the arc length

The method proceeds to find the next equilibrium point from the initial point 0 using the tangent stiffness, $K_{T_{1}}$, as shown in figure 2 . The tangent stiffness matrix is assembled using the nonlinear truss given in (3.28) of section 3 and is defined as the elastic linear stiffness given by (3.6) added to the nonlinear stiffness in (3.26). The sum of the linear elastic and nonlinear matrices produce the global tangent stiffness at point $i$ along the load displacement path of the single-degree-of-freedom system. A new displacement, $\Delta q_{\text {tot }_{i}}$ is calculated from

$$
K_{T_{i}} \Delta q_{\text {tot }_{i}}=\lambda Q
$$

with $\lambda=1$ as before. 


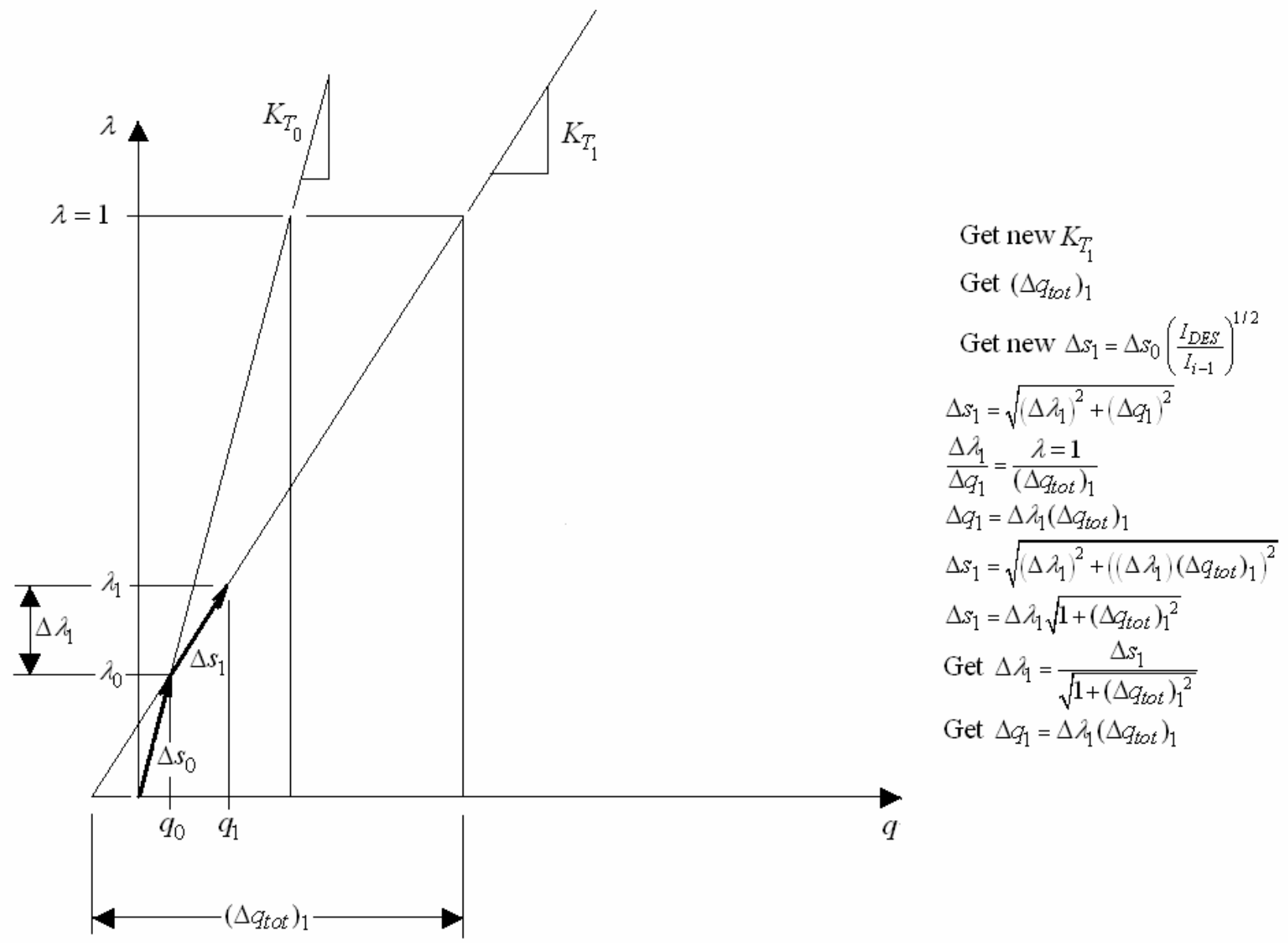

Figure 2. Next iteration

The arc length used by Riks-Wempner is the straight line $\Delta s_{i}$ and is either constant or scaled by the user input using the following

$$
\Delta s_{i}=\Delta s_{i-1}\left(\frac{I_{d e s}}{I_{i-1}}\right)^{\frac{1}{2}}
$$

The user decides on the required number of iterations, $I_{i-1}$, and on the number of desired iterations, $I_{d e s}$. The arc length, $\Delta s_{i}$, is the tangent vector along the equilibrium path and can be calculated as

$$
\Delta s_{0}=\sqrt{\Delta \lambda_{0}^{2}+\Delta q^{T}{ }_{0} \Delta q^{T}}
$$

or using

$$
\Delta s_{0}=\Delta \lambda_{0} \sqrt{1+\Delta q^{T}{ }_{t o t} \Delta q_{t o t}}
$$

The length of the next tangent vector $\Delta s_{1}$ can now be written as 


$$
\Delta s_{1}=\Delta \lambda_{1} \sqrt{1+\left(\Delta q_{t o t}\right)_{1}^{2}}
$$

In this equation the unknown is $\Delta \lambda_{1}$ but can be solved using

$$
\Delta \lambda_{1}=\frac{\Delta s_{1}}{\sqrt{1+\left(\Delta q_{\text {tot }}\right)_{1}^{2}}}
$$

and the incremental displacement found by

$\Delta q_{1}=\Delta \lambda_{1}\left(\Delta q_{\text {tot }}\right)_{1}$

The next step is a test for convergence that first obtains the internal force, $F_{1}$. The residual forces can be calculated as:

$F_{R_{1}}=\lambda_{1} Q-F_{1}$

Here the residual forces are the differences in the internal forces calculated from the equilibrium path and are shown in figure 3.

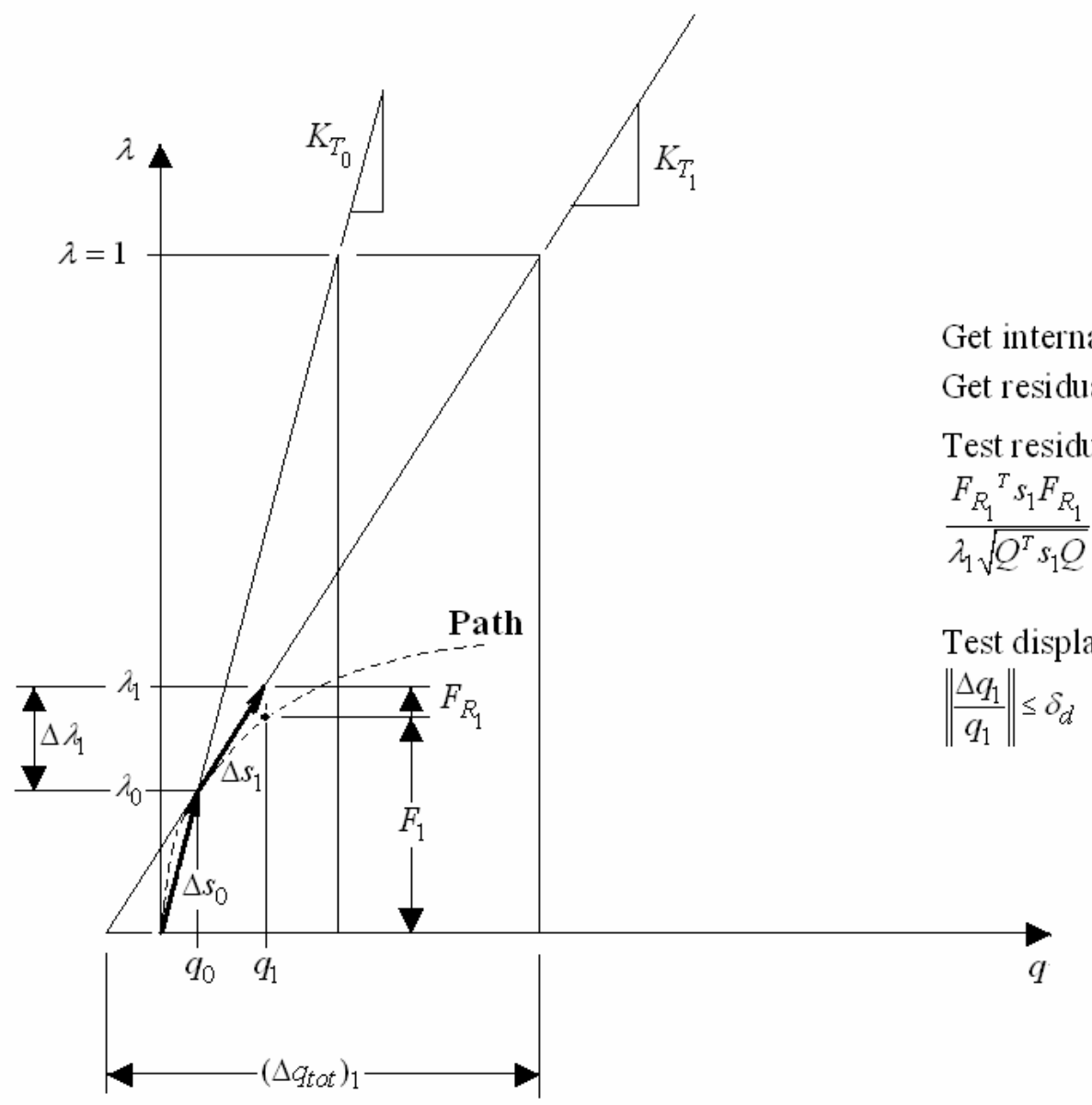

If convergence tests pass, then begin another load step Else

begin iteration on a normal

Figure 3. Convergence tests.

The following is used to test for loads convergence: 
$\frac{F_{R_{1}}{ }^{T} S_{1} F_{R_{1}}}{\lambda_{1} \sqrt{Q^{T} s_{1} Q}} \leq \lambda_{1} Q-\Delta f$

where $\Delta f$ is a user defined tolerance value. If the statement is true then the loads test is successful.

A convergence for displacements is also performed using the ratio $\left\|\frac{\Delta q_{1}}{q_{1}}\right\| \leq \delta_{d}$

with $\delta_{d}$ also a tolerance defined by the user. Once again, if the statement is true then the displacement test is passed.

If both convergence tests are passed then another load step is started with the same procedure being followed as outlined above. If, however, one of the tests fail, then iteration on the normal begins as shown in figure 4 . The objective is to find $q_{2}$ and $\lambda_{2}$ by iterating down the normal from the tangent vector $\Delta s_{1}$. Figure 4 shows the necessary geometry required to start the iteration. A portion of the figure is magnified to help show how the variables are defined.

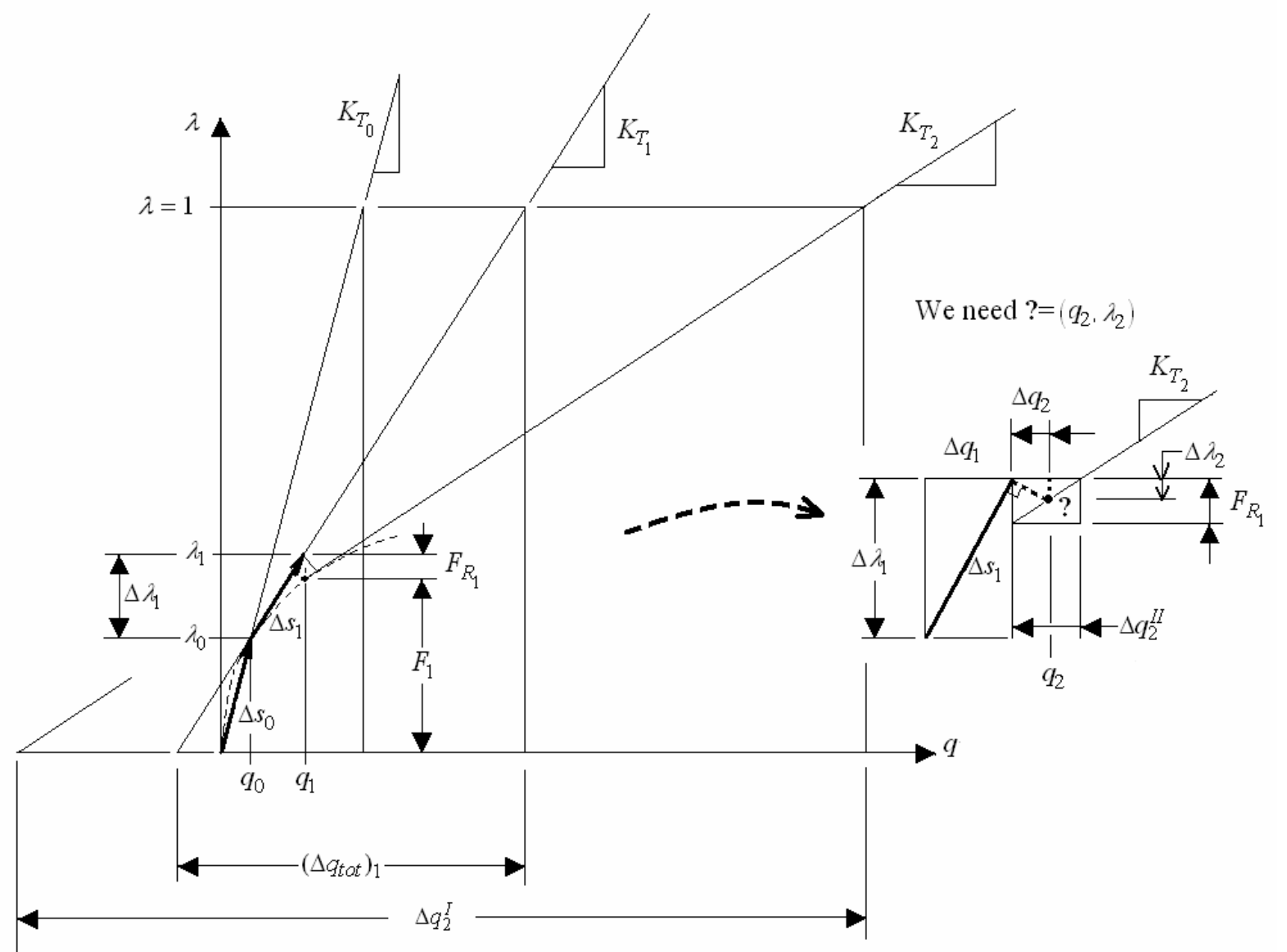

Figure 4. Iteration on a normal.

The normal vector $n_{2}$ is tangent to $\Delta s_{1}$ if 
$\frac{\Delta q_{1}}{\Delta \lambda_{1}}=\frac{\Delta \lambda_{2}}{\Delta q_{2}}$

from similar triangles shown in figure 5. This may also be written as

$\Delta q_{1} \Delta q_{2}-\Delta \lambda_{1} \Delta \lambda_{2}=0$

A new tangent stiffness, $\left[K_{T_{2}}\right]$ is calculated and used in the following

$$
\left[K_{T_{2}}\right] \Delta q_{2}^{I I}=F_{R_{1}}
$$

where all variables are defined by figure 5 .

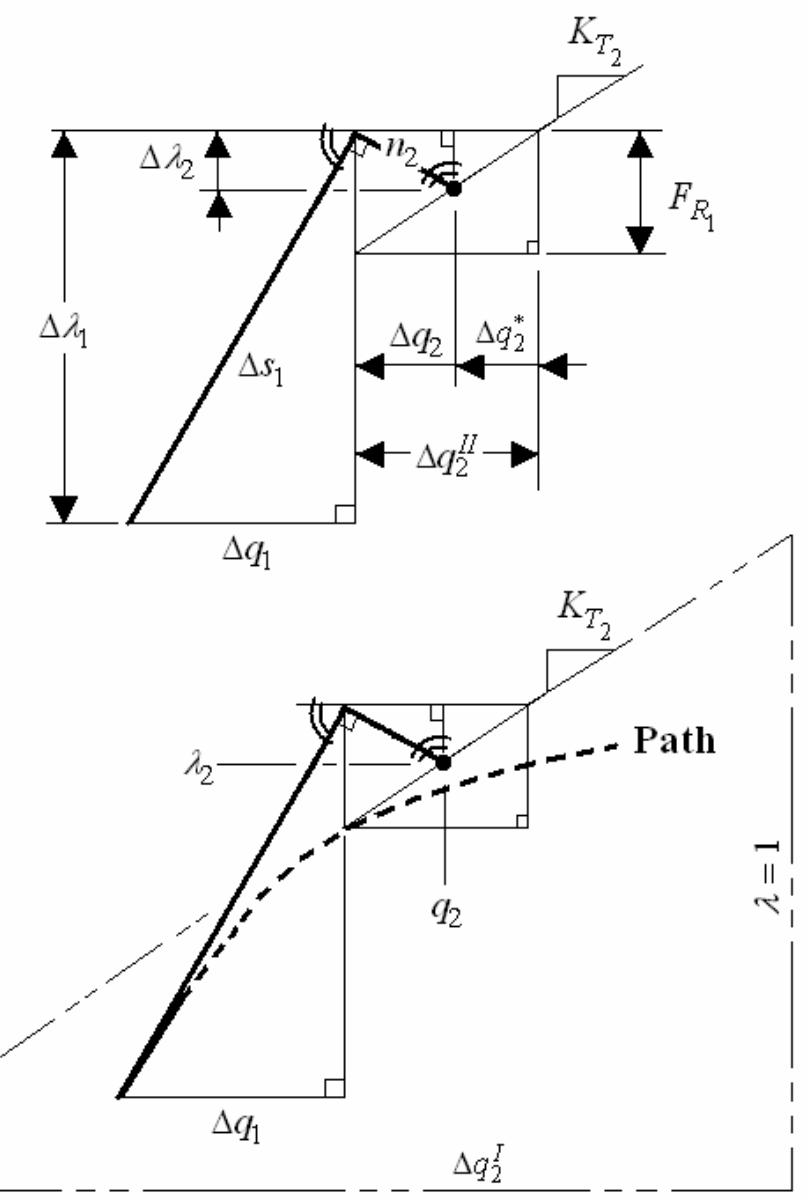

If $n_{2}$ is $\perp \Delta s_{1}$

Use similar triangles:

$\frac{\Delta q_{1}}{\Delta \lambda_{1}}=\frac{\Delta \lambda_{2}}{\Delta q_{2}}$

$\Delta q_{1} \Delta q_{2}-\Delta \lambda_{1} \Delta \lambda_{2}=0 \quad \mathbf{I}$

$\operatorname{Get}\left[K_{T_{2}}\right]$

$\left[K_{T_{2}}\right] \Delta q_{2}^{I I}=F_{R_{1}}$

$\Delta q_{2}^{I I}=\left[K_{T_{2}}\right]^{-1} F_{R_{1}}$

$\Delta q_{2}=\Delta q_{2}^{I I}-\Delta q_{2}^{*}$ II

$\frac{\Delta \lambda_{2}}{\Delta q_{2}^{*}}=\frac{(\lambda=1)}{\Delta q_{2}^{I}}$

$\Delta q_{2}^{*}=\frac{\Delta \lambda_{2} \Delta q_{2}^{I}}{(\lambda=1)}=\Delta \lambda_{2} \Delta q_{2}^{I}$ III

$\Delta q_{2}=\Delta q_{2}^{I I}-\Delta \lambda_{2} \Delta q_{2}^{I}$ III II

$\Delta q_{1}\left(\Delta q_{2}^{I I}-\Delta \lambda_{2} \Delta q_{2}^{I}\right)-\Delta \lambda_{1} \Delta \lambda_{2}=0$ III II I

$\Delta q_{1} \Delta q_{2}^{I I}-\Delta q_{1}\left(\Delta \lambda_{2} \Delta q_{2}^{I}\right)-\Delta \lambda_{1} \Delta \lambda_{2}=0$

$\Delta q_{1} \Delta q_{2}^{I I}-\Delta \lambda_{2}\left(\Delta q_{1} \Delta q_{2}^{I}+\Delta \lambda_{1}\right)=0$

$\Delta \lambda_{2}=\frac{\Delta q_{1} \Delta q_{2}^{I I}}{\left(\Delta q_{1} \Delta q_{2}^{I}+\Delta \lambda_{1}\right)}$

$\lambda_{2}=\lambda_{0}+\Delta \lambda_{1}+\Delta \lambda_{2}$
$q_{2}=q_{0}+\Delta q_{1}+\Delta q_{2}$

Figure 5. Details of iterating on a normal.

Equation (2.17) may be rewritten as

$\Delta q_{2}^{I I}=\left[K_{T_{2}}\right]^{-1} F_{R_{1}}$

The incremental displacement shown in figure 5 can be expressed as

$\Delta q_{2}=\Delta q_{2}^{I I}-\Delta q_{2}^{*}$

Now, from similar triangles the following is obtained 
$\frac{\Delta \lambda_{2}}{\Delta q_{2}^{*}}=\frac{(\lambda=1)}{\Delta q_{2}^{I}}$

Rearranging terms to obtain

$\Delta q_{2}^{*}=\frac{\Delta \lambda_{2} \Delta q_{2}^{I}}{(\lambda=1)}=\Delta \lambda_{2} \Delta q_{2}^{I}$

Combining equations (2.19) and (2.21) will create

$\Delta q_{2}=\Delta q_{2}^{I I}-\Delta \lambda_{2} \Delta q_{2}^{I}$

Now equation (2.16) is combined with (2.22) to obtain

$\Delta q_{1}\left(\Delta q_{2}^{I I}-\Delta \lambda_{2} \Delta q_{2}^{I}\right)-\Delta \lambda_{1} \Delta \lambda_{2}=0$

that may be reduced to

$\Delta \lambda_{2}=\frac{\Delta q_{1} \Delta q_{2}^{I I}}{\left(\Delta q_{1} \Delta q_{2}^{I}+\Delta \lambda_{1}\right)}$

which is added to the previous load increments as follows

$\lambda_{2}=\lambda_{0}+\Delta \lambda_{1}+\Delta \lambda_{2}$

Likewise, the displacements increments are summed using (2.22) to give

$q_{2}=q_{0}+\Delta q_{1}+\Delta q_{2}$

Equations (2.24) and (2.25) represent the load and displacement at location 2 along the equilibrium path. A convergence check is performed similar to figure 3 and equations (2.12) through (2.14). If the test is passed then a new load increment starts using the tangent vector of equation (2.9). If the tests fail then iteration down the normal vector $n$ is started as shown in figure 6 . The process continues until the convergence tests for load and displacements are both passed. 


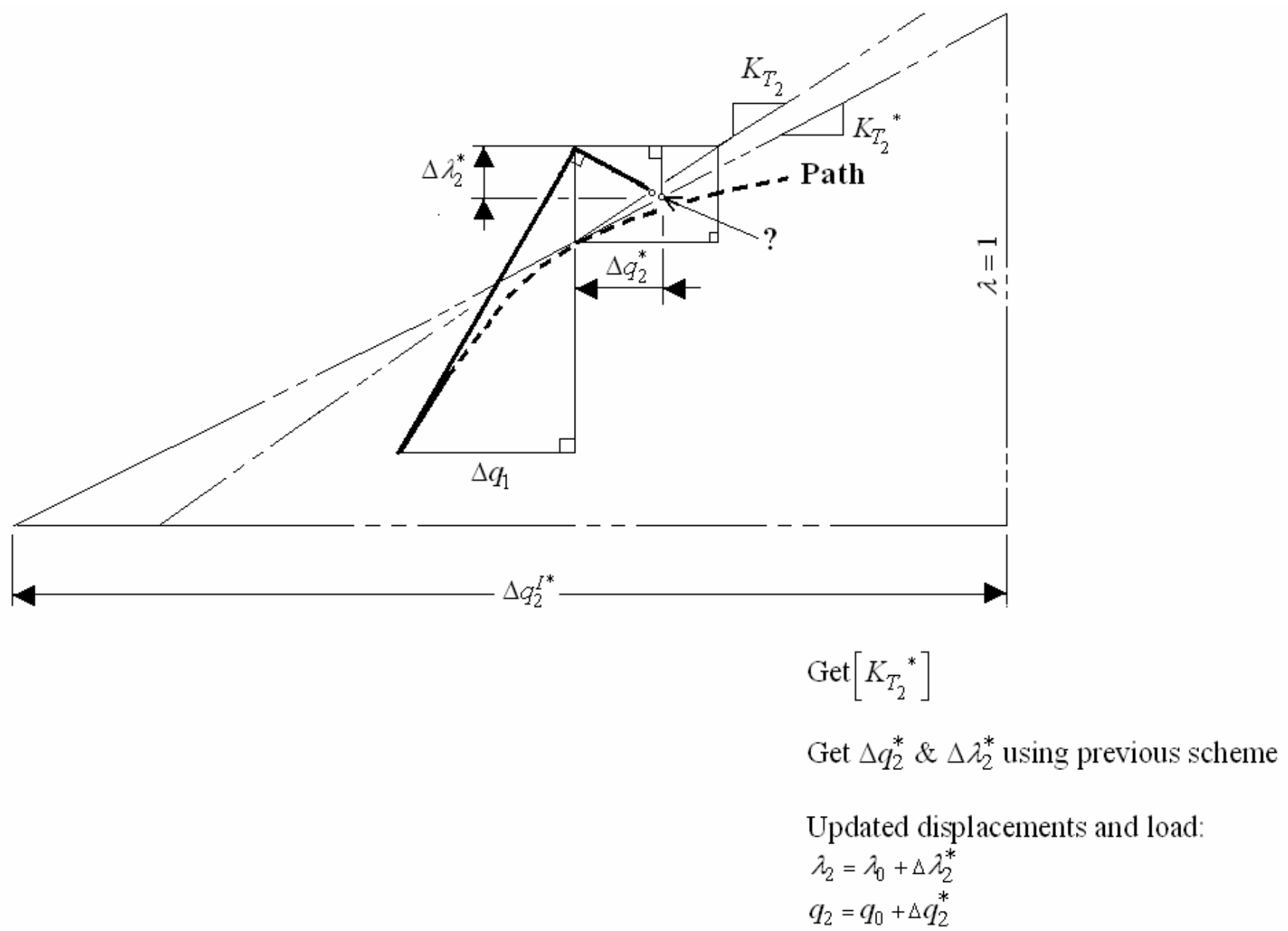

Figure 6. Continuing iteration on normal

\section{Derivation of 3-D Geometrically Nonlinear Truss Element Stiffness}

Trusses undergoing large deflections must be analyzed for the deformed geometry of the structure. The linear equation

$$
F=[K] u
$$

that relates the applied force $F$ with the truss element stiffness $[K]$ and displacements $u$ must be modified to account for changes in nodal geometry as the load is applied. The standard stiffness elastic stiffness matrix $[K]_{E}$ will be modified by the addition of a geometric stiffness matrix $[K]_{G}$ that depends on the geometry and initial internal forces. The elastic and geometric matrices can be written as:

$$
[K]=[K]_{E}+[K]_{G}
$$

It should be noted here that efficient sparse assembly procedures [12] have been employed in 
Eq. (3.2), while efficient sparse reordering algorithms (to minimize fill-in terms, during factorization phase) and sparse solver (with unrolling strategies [12] have been utilized in Eq.

Nonlinear analysis is usually divided into a sequence of load steps with the displacements being solved after each load increment. The nonlinear stiffness is then updated with the displacement and internal force values from the previous step. A discussion of the nonlinear truss element used in the finite element program in this study follows.

The following definitions are used to derive the elastic truss stiffness with the variables defined in figure 7. The change in displacements for an $i^{\text {th }}$ truss can be written as

$\Delta X^{(i)}=x_{2}-x_{1}+\Delta x_{2}-\Delta x_{1}$

$\Delta Y^{(i)}=y_{2}-y_{1}+\Delta y_{2}-\Delta y_{1}$

$\Delta Z^{(i)}=z_{2}-z_{1}+\Delta z_{2}-\Delta z_{1}$

where $\Delta x_{1}, \Delta y_{1}, \Delta z_{1}$ are the displacements at end 1 and $\Delta x_{2}, \Delta y_{2}, \Delta z_{2}$ are the displacements at end 2. The global nodal coordinates are given as $x_{1}, y_{1}, z_{1}$ at end 1 and $x_{2}, y_{2}, z_{2}$ for end 2. The original length is $L^{(i)}$ and the new length is given as $\Delta L^{(i)}$.

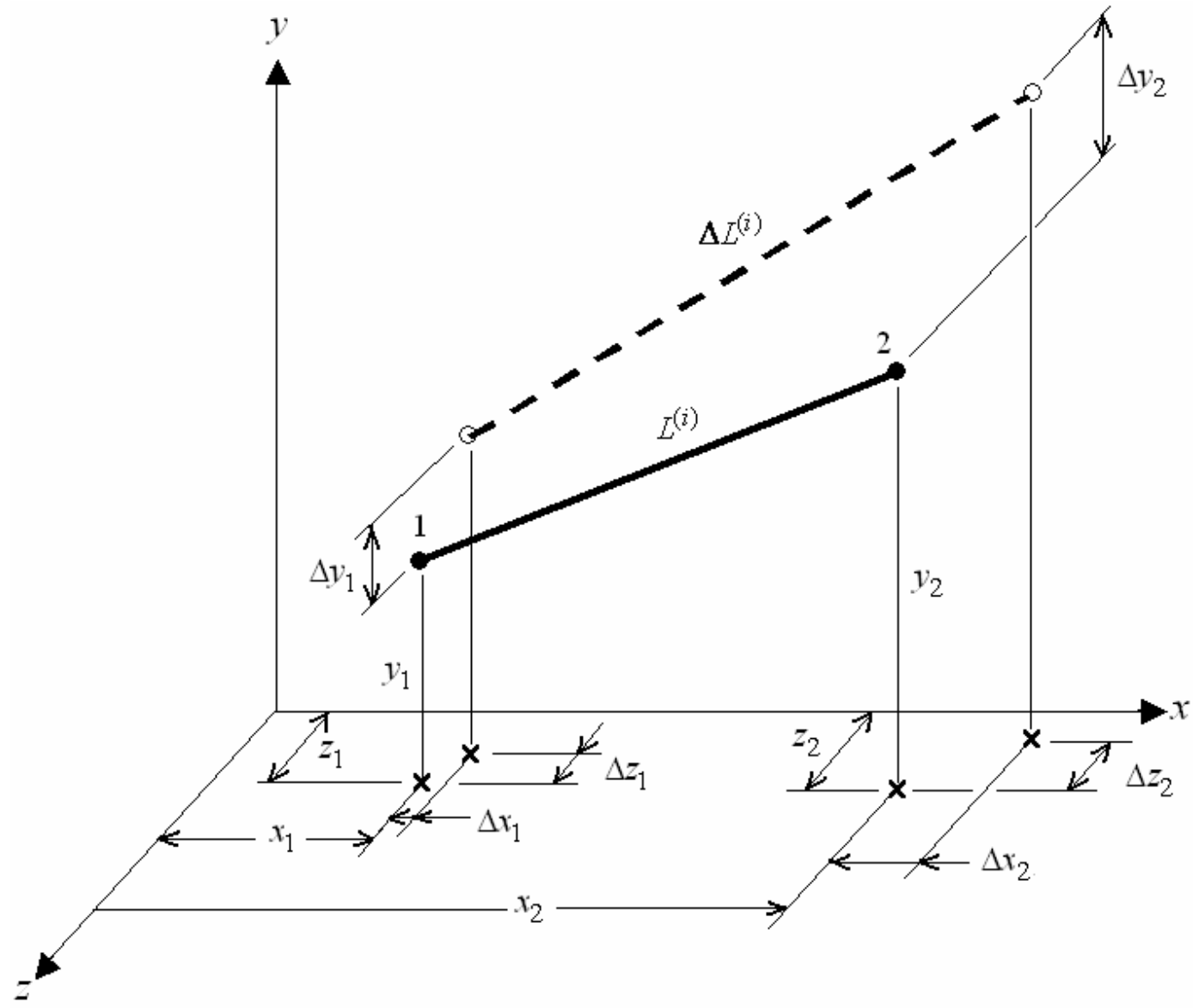

Figure 7. Nonlinear 3d Truss variables 
The matrix [esm] shown below is the normal elastic stiffness matrix for a three dimensional truss defined using variables for easier computer programming.

$$
[e s m]=C O N 1\left[\begin{array}{llllll}
C(1) C(1) & C(1) C(2) & C(1) C(3) & C(1) C(4) & C(1) C(5) & C(1) C(6) \\
& C(2) C(2) & C(2) C(3) & C(2) C(4) & C(2) C(5) & C(2) C(6) \\
& & C(3) C(3) & C(3) C(4) & C(3) C(5) & C(3) C(6) \\
& & & C(4) C(4) & C(4) C(5) & C(4) C(6) \\
& & & & C(5) C(5) & C(5) C(6) \\
& & & & & C(6) C(6)
\end{array}\right]
$$

where the following are defined as:

$$
\begin{aligned}
& X 21 D=\text { deltax }_{i} \\
& Y 21 D=\text { delta }_{i} \\
& Z 21 D=\text { deltaz }_{i} \\
& C(1)=-x 21 D \\
& C(2)=-Y 21 D \\
& C(3)=-Z 21 D \\
& C(4)=-C(1) \\
& C(5)=-C(2) \\
& C(6)=-C(3) \\
& C O N 1=\frac{E A}{x l^{3}}
\end{aligned}
$$

with $E A$ being Young's modulus multiplied with the bar cross section. The nonlinear geometric stiffness will now be defined and added to the elastic stiffness (3.6).

\section{Element internal force}

The internal forces in the truss element are required for the geometric stiffness and will be defined using matrix notation. The element strain formulation uses a constant cross sectional area and assumes the length/area of the truss will remain large. The strain energy or work done is $1 / 2$ the nodal forces multiplied by the corresponding deflections.

The internal force in the truss will now be defined to include nonlinear effects.

The force will be axial and is needed in updating the nonlinear stiffness matrix.

Defining the force in matrix notation is necessary for efficient computer programming. The truss length from incremental displacements may be written as

$x l i^{2}=\left(\vec{x}_{21}+\vec{p}_{21}\right)^{T}\left(\vec{x}_{21}+\vec{p}_{21}\right)$

where 
$\vec{p}_{21}=\left[\begin{array}{c}\left(u x_{2}-u x_{1}\right) \\ \left(u y_{2}-u y_{1}\right) \\ \left(u z_{2}-u z_{1}\right)\end{array}\right] \quad$ and $\quad \overrightarrow{x l}_{21}=\left[\begin{array}{c}\left(x_{2}-x_{1}\right) \\ \left(y_{2}-y_{1}\right) \\ \left(z_{2}-z_{1}\right)\end{array}\right]$

with

$u x_{21}=u x_{2}-u x_{1}$

$u y_{21}=u y_{2}-u y_{1}$

$u z_{21}=u z_{2}-u z_{1}$

Now using the strain equation

$\varepsilon=\frac{x l i^{2}-x l^{2}}{2 x l^{2}}$

and substituting in the original and new truss lengths in matrix form will give

$\varepsilon=\frac{\left(\overrightarrow{x l}_{21}+\vec{p}_{21}\right)^{T}\left(\overrightarrow{x l}_{21}+\vec{p}_{21}\right)-\left(\overrightarrow{x l}_{21}^{T} \vec{x}_{21}\right)}{2 \overrightarrow{x l}_{21}^{T} \overrightarrow{x l}_{21}}$

This may be simplified in matrix notation written as

$$
\varepsilon=\overrightarrow{x l}_{21}^{T} \vec{p}_{21}+\frac{1}{2} \vec{p}_{21}^{T} \vec{p}_{21}
$$

The first portion of (3.24) represents the linear strain and the last part is the nonlinear contribution. The internal force in the truss is then:

$$
A N=E A \varepsilon
$$

and the geometric stiffness can now be defined as:

$$
K_{G}=\left[\begin{array}{cccccc}
\frac{A N}{x l} & 0 & 0 & -\frac{A N}{x l} & 0 & 0 \\
& \frac{A N}{x l} & 0 & 0 & -\frac{A N}{x l} & 0 \\
& \frac{A N}{x l} & 0 & 0 & -\frac{A N}{x l} \\
& & \frac{A N}{x l} & 0 & 0 \\
& & & \frac{A N}{x l} & -\frac{A N}{x l} \\
& & & & \frac{A N}{x l}
\end{array}\right]
$$


where

$$
\frac{A N}{x l}=\frac{E A}{x l}\left[\frac{\left(u x_{2}-u x_{1}\right)}{x l}+\frac{\left(u y_{2}-u y_{1}\right)}{x l}+\frac{\left(u z_{2}-u z_{1}\right)}{x l}+\frac{\left(u x_{2}-u x_{1}\right)^{2}}{2 x l^{2}}+\frac{\left(u y_{2}-u y_{1}\right)^{2}}{2 x l^{2}}+\frac{\left(u z_{2}-u z_{1}\right)^{2}}{2 x l^{2}}\right]
$$

The total nonlinear 3D truss element stiffness can now be written by adding the corresponding members of 3.27 with 3.16 that reduces until the final tangent stiffness becomes:

$$
K_{T}=\frac{E A}{x l}\left[1+\frac{\left(3\left(u x_{2}-u x_{1}\right)\right)}{x l}+\frac{\left(3\left(u y_{2}-u y_{1}\right)\right)}{x l}+\frac{\left(3\left(u z_{2}-u z_{1}\right)\right)}{x l}+\frac{3\left(\left(u x_{2}-u x_{1}\right)^{2}\right)}{2 x l^{2}}+\frac{3\left(\left(u y_{2}-u y_{1}\right)^{2}\right)}{2 x l^{2}}+\frac{3\left(\left(u z_{2}-u z_{1}\right)^{2}\right)}{2 x l^{2}}\right]
$$

This is the tangent stiffness used for a nonlinear truss element in the developed finite element algorithm. The truss element's performance was tested against snap through and snap back problems found in Crisfield [2,3]. Criteria for a well behaved element were its ability to accurately trace an equilibrium path and to reach and pass critical limit points within an arc length solution.

\section{Derivations of Optimum Nonlinear Strain Energy Density Formulas}

Optimizing the weight of truss structures undergoing large displacements requires analysis methods that accurately trace the equilibrium path and identifies critical limit points. Most "shallow type" space truss designs will have snap through response curves similar to figure 8 . Depending on the geometry of the design, the structure may have a snap back path or may respond with bifurcation buckling. These stability response types are due to the level of nonlinearity of the geometry in the design and the applied loading. Imperfections in the truss geometry can also contribute to a reduced load carrying ability of a system. The investigation of random initial imperfections for frame-type structures that exhibit instability at limit points was discussed by Warren in [17]. He used an arc length approach to trace the nonlinear equilibrium paths of simple benchmark examples. Khot [7] explains the reduction in load capacity is primarily from the nonlinear response of a system and secondary from geometry imperfections. Verification problems from Crisfield $[2,3]$ show the snap through and snapback behavior of several shallow truss designs. Achieving a minimum weight design of shallow trusses similar the design examples from Crisfield will require limit point stability constraints that can maximize critical load.

The discussion of using the strain energy density as an indicator of an optimal design starts with explaining strain and potential energy in truss elements. The optimization problem can then be defined with a recurrence formula developed to update the design variable, in this case the cross sectional area of the member.

\section{Strain Energy Density}

The strain in a truss element can be expressed as

$\varepsilon=\frac{x l i-x l}{x l}$ 
where $x l$ is the original length and $x l i$ is the new length as used in chapter 2 . The element's length change may be large, however, the strains are considered small enough to allow a linear stress strain relationship. The work done by external forces causing deformation is stored within the truss in the form of strain energy. In a load displacement profile, the area under the curve is the strain energy (SE) and can be written as

$$
S E=\frac{1}{2} P \Delta
$$

where $P$ is the internal load and $\Delta$ is the axial displacement.

Substituting $P=\frac{A E \Delta}{x l}$ for a truss element will give

$S E=\frac{1}{2} \frac{A E}{x l} \Delta^{2}$

Here $A$ is the truss cross sectional area and $E$ is Young's modulus.

Upon further manipulation with $\varepsilon^{2}=\frac{(x l i-x l)^{2}}{x l^{2}}=\frac{\Delta^{2}}{x l^{2}}$, the strain energy for an $i$ th element can be expressed as

$S E_{i}=\frac{1}{2} E_{i} A_{i} x l_{i} \varepsilon_{i}^{2}$

Strain energy density (SED) is the amount of strain energy per unit volume. It can be found by dividing the strain energy by the volume of an element and can be written as

$$
S E D_{i}=\frac{S E_{i}}{x l_{i} A_{i}}
$$

This relationship is modified for use in an optimum criterion by dividing the strain energy by the mass density $\rho_{i}$ for an ith element that gives

$$
S E D_{i}=\frac{S E_{i}}{\rho_{i} x l_{i} A_{i}}
$$

In order to further develop an optimality criterion for stability, a discussion of potential energy follows.

\section{Optimality Criterion}

The minimum weight optimization of a truss system is defined as:

$$
W=\sum_{i=1}^{n} \rho_{i} A_{i} L_{i}
$$

where $\rho_{i}$ is the material density of the $i$ th element, $A_{i}$ is the $i$ th element cross sectional area and $L_{i}$ is the new deformed length of the $i$ th element with $n$ number of elements. 
The formal optimization problem can be defined as:

Find $\quad\left\{A_{i}\right\}$

to minimize $\quad \sum_{i=1}^{n} \rho_{i} A_{i} L_{i}$

subject to $\quad P E-\overline{P E}=0$

This minimum weight equation is subject to the equality constraint

$g=P E-\overline{P E}=0$

where $P E$ is the total potential energy of a truss system and $\overline{P E}$ is the target total potential energy of the optimal design at the critical nonlinear limit load. This equality constraint has the smallest cross sectional area capable of sustaining an applied load just before the structure becomes unstable. This places a constraint on the limit load that is the same as constraining the total potential energy of the structure. For multiple members the potential energy may be written as

$P E=\sum_{i=1}^{\text {ele }} S E_{i}-\sum_{j=1}^{\text {nodes }} W_{j}$

where $S E_{i}$ is the strain energy in the $i$ th element and $W_{j}$ is the work produced from an external force. The relationship can also be written for a structural system consisting of $n$ number of truss elements as:

$P E=\sum_{i=1}^{n} S E_{i}-\vec{u}^{T} \vec{F}$

with $S E_{i}$ identifying the strain energy in the $i$ th element, $\vec{u}^{T}$ a vector transpose of the global displacements and $\vec{F}$ being the applied design load vector.

The constrained minimization of equation (4.7) with the equality constraint is summarized as:

Find $\left\{A_{i}\right\}$

to minimize $\sum_{i=1}^{n} \rho_{i} A_{i} x l_{i}$

subject to

$$
P E-\overline{P E}=0
$$

The first step to solving the nonlinear constrained optimal problem and deriving an update scheme for the area is to form the Lagrangian. The method will answer the question "How do I minimize the strain energy density while making sure the structure will support a 
load without becoming unstable?" It is the basic tool used in nonlinear constrained optimization problems.

Lagrange developed the tool for solving optimality problems by developing the Lagrangian function written as

$L=f(x)+\sum_{j=1}^{m} \gamma_{j} h_{j}(x)$

for finding $\quad x \in R^{n}$

to minimize $f(x)$

subject to $\quad h_{j}(x)=0 \quad j=1,2, \ldots, m$

where $\gamma_{j}$ is called the Lagrange multiplier for the solution (x) that lies in some domain.

Lagrange multipliers find the largest maximum or smallest minimum of a function of several variables subject to one or more constraints. This method reduces a problem in $n$ variables with $k$ constraints to a solvable problem in $n+k$ variables with no constraints. The method introduces a new unknown scalar variable, the Lagrange multiplier, for each constraint and forms a linear combination involving the multipliers as coefficients. The Lagrange multiplier can be thought of as how strong the constraint is working to get a point to a maximum or minimum. The objective is to find the conditions, for some implicit function, so that the derivative in terms of the independent variables of a function equals zero for some set of inputs. The point $x$ is stationary in space if

$\frac{\delta L}{\delta x_{i}}=0=\frac{\delta f}{\delta x_{i}}+\sum_{j=1}^{m} \gamma_{j} \frac{\delta h_{j}(x)}{\delta x_{i}} \quad i=1,2, \ldots, n$

and

$h_{j}(x)=0 \quad j=1,2, \ldots, m$

A stationary point merely means that the solution (x) causes the derivative of a function to equal zero. This is equivalent to saying it's where the function stops increasing or decreasing. In the case presented in this paper, the solution (x) is the value of the load that causes the tangent at that point to be parallel to the $\mathrm{x}$-axis. This is also the point of minimum strain energy density for all members along their equilibrium path. Using this formulation and applying it to minimizing weight in trusses, the Lagrangian becomes

$L=\sum_{i=1}^{n} \rho_{i} A_{i} x l_{i}-\gamma(P E-\overline{P E})$

where $\gamma$ is the Lagrange multiplier and $(P E-\overline{P E})$ is the difference between the total

potential energy of the system $P E$ and the target potential energy $\overline{P E}$ of the optimal design at the critical limit load. We desire this difference in potential energy to be a minimum.

Differentiating with respect to $A_{i}$ gives 
$\rho_{i} x l_{i}-\gamma_{1}\left(\frac{\partial P E}{\partial A_{i}}+\sum_{j=1}^{d o f} \frac{\partial P E}{\partial u_{j}} \frac{\partial u_{j}}{\partial A_{i}}\right) \quad i=1,2, \ldots, n$

The potential energy is a function of the set of displacements. If these displacements behave to place the structure in stable equilibrium then the total potential energy has a "stationary" value and the following can be written

$\frac{\partial P E}{\partial u}=\frac{\partial S E}{\partial u}-F=0$

This is the partial of the potential energy from (4.10). Using the zero statement of (4.22) with (4.21) the following simplified form is achieved:

$\rho_{i} x l_{i}-\gamma_{1}\left(\frac{\partial P E}{\partial A_{i}}\right)=0$

Consider now that the potential energy in (4.10) as function of the area of the section. A change in the potential energy with respect to a change in the area can be written as

$\frac{\partial P E}{\partial A_{i}}=\frac{\partial S E_{i}}{\partial A_{i}}-\frac{\partial u_{i} F}{\partial A_{i}}$

This is equal to

$\frac{\partial P E}{\partial A_{i}}=\frac{S E_{i}}{A_{i}}$

This is possible because of the definition of strain energy in (4.4)

Placing (4.25) into (4.23) yields

$\rho_{i} x l_{i}-\gamma_{1}\left(\frac{S E_{i}}{A_{i}}\right)=0$

This can be rewritten as

$\frac{\gamma_{1}}{\rho_{i} x l_{i}}\left(\frac{S E_{i}}{A_{i}}\right)=1$

Using the definition of strain energy density this equation can be given as the strain energy density optimality criterion of

$\gamma_{1}\left(S E D_{i}\right)=1$

A recurrence formula for updating the area design variable is found by multiplying this formula by $\left(A_{i}\right)^{\beta}$ to give

$$
\left(A_{i}\right)^{\beta}=\left(A_{i}\right)^{\beta}\left(\gamma_{1}\right)\left(S E D_{i}\right)
$$

where $\beta$ is a parameter that controls the step size and convergence rate.

Now taking the $\beta$ th root on each side

$\left(A_{i}\right)_{i t e r+1}=\left(A_{i}\right)_{i t e r}\left(\gamma_{1}\right)\left(S E D_{i}\right)^{1 / \beta}$

gives the updated area for the next iteration. The use of this equation within the arc length method will set $1 / \beta$ equal to the load increment multiplier $\lambda_{i}$ as shown in figure 1 . The Lagrange multiplier $\gamma$ in equation (4.30) is set equal to 


$$
\gamma_{1}=\frac{\sum_{i=1}^{n} S E D}{\sum_{i=1}^{n} S E D^{2}}
$$

and is placed into equation (4.30) along with $\lambda_{i}$ to obtain

$$
\left(A_{i}\right)_{i t e r+1}=\left(A_{i}\right)_{i t e r}\left(\frac{\sum_{i=1}^{n} S E D}{\sum_{i=1}^{n} S E D^{2}}\right)\left(S E D_{i}\right)^{\lambda}
$$

This is the recurrence relation used to update the design variable $A_{i}$ at the end of each converged arc length iteration. This is a type of difference equation that defines each term of the sequence as a function of the preceding terms. Equation (4.32) gives a new $A_{i}$ based on the strain energy density difference in all the members. As an optimal solution is approached the SED's will become equal within a user specified difference allowable.

\section{Design Variable Scaling}

The arc length method is used to both solve the nonlinear structural system and also as an update indicator used in monitoring the SED in the system's truss elements. As the equilibrium path is defined, parameters of the arc length are monitored and used to update the recurrence equation. The approach allows for updating the truss stiffness after each run through the arc length solver. As a critical limit point is approached, the slope of the tangent to the equilibrium path will approach zero. The ideal solution would place the critical limit load when the slope goes to zero and all the member strain energy densities become equal.

A recurrence relation is used to update the design variables, in this case the cross sectional areas of the truss members. Recurrence equations are used in iterative processes to keep track and adjust variables in optimizations. Formulation of the updating recurrence relation relies on information from the arc length parameters. One of those parameters is the incremental external load multiplier. The multiplier picked to update the recurrence equation depends on what phase the arc length solver is processing. The solver has three phases with each checking the difference between the present total external load multiplier and the next load multiplier. This is shown beside the "call rikarc" in the flowchart of figure 9. The checks indicate how close the load is to reaching a limit point and if the arc length is becoming horizontal with decreasing slope.

A new parameter, called the scale strain parameter (SSP), was developed that used the size of the incremental external load at the end of each arc length iteration. The value of the SSP depends on the checks performed above and shown in figure 9. The three possible values of the scale strain parameter are

$$
\mathrm{SSP}=\lambda_{0}+\Delta \lambda_{0}, \lambda_{i}+\Delta \lambda_{i} \text { or } \lambda_{k}+\Delta \lambda_{k}
$$


where $\Delta \lambda_{0}, \Delta \lambda_{i}$ or $\Delta \lambda_{k}$ are the incremental load multipliers used along the load path. The initial load increment is $\Delta \lambda_{0}=d l 0$ and is supplied by the user at the start of the arc length procedure as shown in figure 1 . The value $\lambda_{0}$ is a preload placed on the structure but is zero for the problems used in this paper. Figure 8 shows the other two incremental multipliers as: $\Delta \lambda_{i}=d l i$....the incremental external multiplier at step i $\Delta \lambda_{k}=d l k \ldots .$. the incremental external multiplier at step $\mathrm{k}$
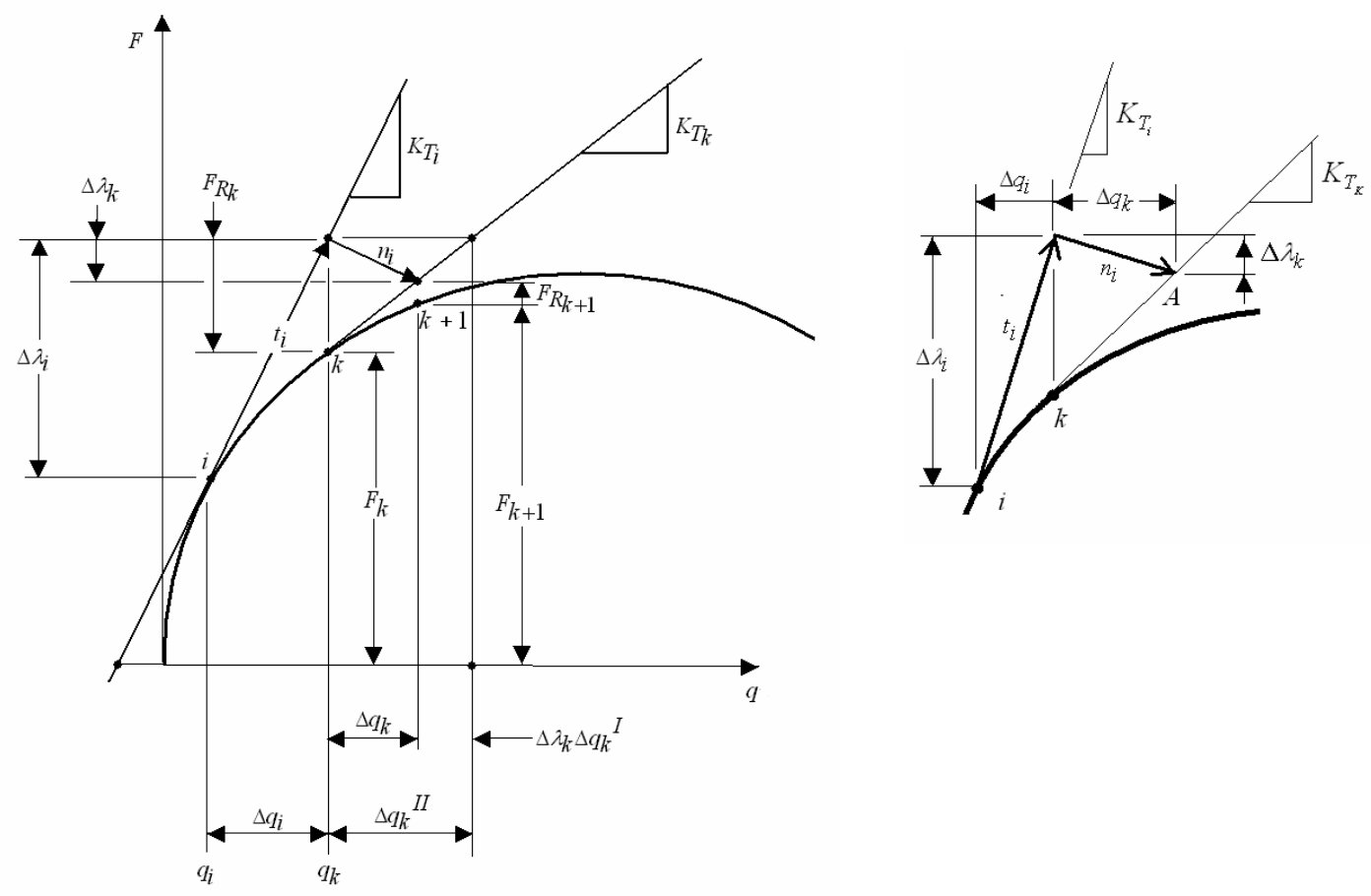

Figure 8. Riks-Wempner arc length method on a normal plane for a single-degree-of-freedom system.

The new recurrence formula for scaling the design variable is taken from (4.32) and can be written using the SSP as:

SEDscale $=(($ SEDtotal $/$ SEDtotal $) * S E D(i)) * * S S P$

where:

SEDscale is the strain energy density scaling factor

SEDtotal is the total strain energy density

SEDtotal2 is SEDtotal ${ }^{\wedge} 2$

$S E D(i)$ is the strain energy density in the $i$ th member and

$S S P$ is the new scale strain parameter

$d S E D t o l=1.0 \mathrm{~d}-3$ user given differences between all elements SED

The flowchart shown in figure 9 outlines the updating scheme of the area design variable, propsect. Immediately after a solution is found in the arc length solver, the elemental strain 
energies are calculated and compared with the strain energy density tolerance, dSEDtol. If all members are within the tolerance then the solution is the optimal. If one member fails the tolerance test then a new SSP is calculated from the initial multiplier, $d l 0$, or the incremental external multipliers $d l i$ and $d l k$.

The strain energy density check is shown in the flowchart of figure 9 and graphically explained in figure 8. Each user defined tolerance depends on the type of model, level of fidelity of the user wishes in weight reduction and how the structure was initially modeled. The whole structure can be optimized for weight or truss members may be grouped together for sizing. If the absolute SED difference is below the tolerance, then the solution is considered at optimum. A minimum area is used to avoid zero area trusses. A zero area indicates a truss member that is necessary for a stable structure but carries no load. The minimum area check is made to assure member sizes are greater than a user provided area.

Another check of all the $S E D$ 's is made using the user provided $d S E D t o l$. Only solid rods are used in this work but any section could be used. The new strain energy densities are then computed using the updated areas.

The strategies explained in using the recurrence formula will be demonstrated using example problems that are solved using a nonlinear solution in Nastran. Results of nonlinear structures published in numerous references will also be used to verify the Hrinda algorithm using the strain energy density and arc length procedure. 


\section{Optimizer Flowchart}

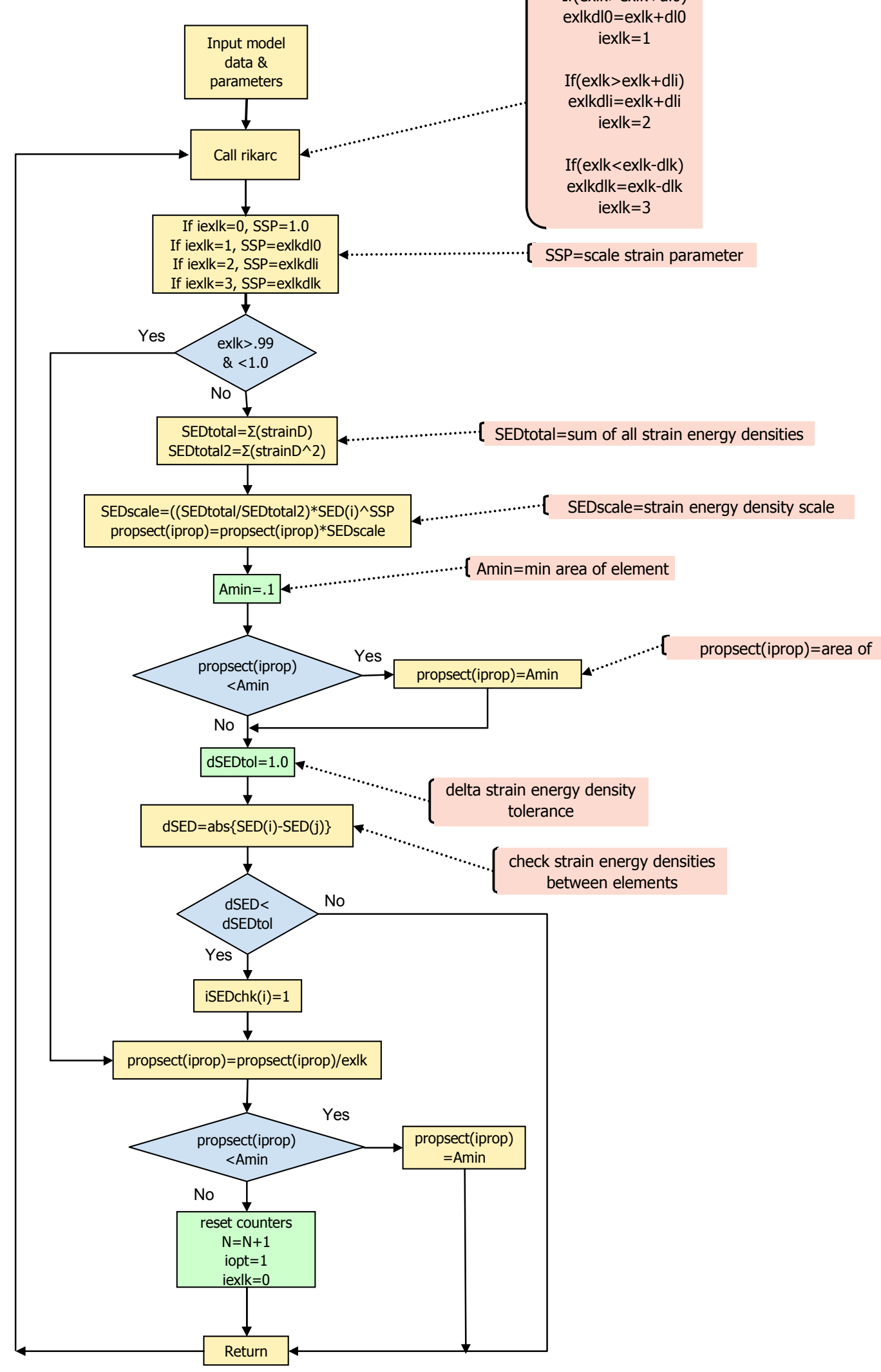

Figure 9. Flowchart for the Hrinda optimizer program. 


\section{Verification Examples of Optimized Trusses}

The following example problems are used to verify the proposed nonlinear optimization method. All of the examples attempt to find the minimum weight of truss areas to support an applied load without buckling. The problems are shallow trusses that have critical limit points and are take from Khot [7]. The first two problems solve a two element, 2D system. A vertical load is applied at the mid point with pinned end conditions. The next problem is a four element system that has four different lengths with four cross sections to be optimized. A larger star dome problem is then presented similar to the Crisfield star dome in volume two [4]. This problem was minimized using four cross sectional area groups related to the layout of the design. The last problem is a large shallow truss that is more practical to space trusses. The problem allows for each of the members to be solved for a unique design variable. Here the system has 13 cross sections sized to support a vertical load without snapping through. The solutions found in all examples were compared to those published by Khot as well as with Nastran fea. The areas found by the proposed technique were used in the Nastran models. Here the displacements of the Nastran solutions were checked against those found by the proposed algorithm.

\section{Example 1. Two Member Symmetric Truss}

The first example used to help verify the Hrinda optimization algorithm is a two member symmetric shallow truss with a 200. 1b. concentrated load as shown in figure 10 . The problem is taken from Khot [7] and is optimized to find the minimum sectional area that could support the 200. lb. load without a sudden strain energy release and the structure snapping through. All degree-of-freedoms were constrained except at the center node.

Two different initial areas of 3.0 sq. inch and 5.0 sq. inch were chosen to test the algorithms ability to find unique areas for each member. Figure 11 shows the initial and final strain energy density distributions in the two elements. The SED in the members became equal at .88199 making their final relative SED equal to 1.0 indicating an optimal design. Both members were sized to equal areas of $6.499 \mathrm{sq}$. inch. that was slightly larger then the results found by Khot [7] of 6.4977 sq. inch for both members. The total weight summary of the members after each iteration is given in figure 12 .

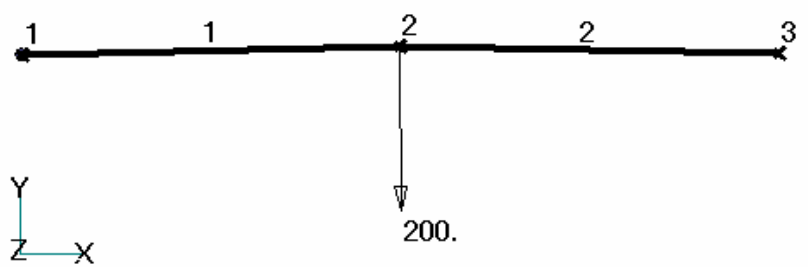

Figure 10. Two element symmetric truss with $200 \mathrm{lbs}$. apex load.

\begin{tabular}{|c|c|c|c|c|c|c|}
\hline Element & $\begin{array}{c}\text { Initial Area } \\
\left(\text { in }^{2}\right)\end{array}$ & Initial SED & $\begin{array}{c}\text { Initial Relative } \\
\text { SED }\end{array}$ & $\begin{array}{c}\text { Final } \\
\text { SED }\end{array}$ & $\begin{array}{c}\text { Final Relative } \\
\text { SED }\end{array}$ & $\begin{array}{c}\text { Final Area } \\
\left(\text { in }^{2}\right)\end{array}$ \\
\hline 1 & 3.00 & 1.38806 & 1.00000 & 0.88199 & 1.00000 & 6.4989 \\
\hline 2 & 5.00 & 0.49960 & 0.35993 & 0.88199 & 1.00000 & 6.4989 \\
\hline
\end{tabular}

Figure 11. Strain energy density distribution of two element symmetric truss. 


\begin{tabular}{|c|c|}
\hline Iteration & Weight (lbs.) \\
\hline 1 & $0.100020 \mathrm{E}+03$ \\
\hline 2 & $0.803313 \mathrm{E}+02$ \\
\hline 3 & $0.799023 \mathrm{E}+02$ \\
\hline 4 & $0.162505 \mathrm{E}+03$ \\
\hline
\end{tabular}

Figure 12. Iteration weight history of two element symmetric truss.

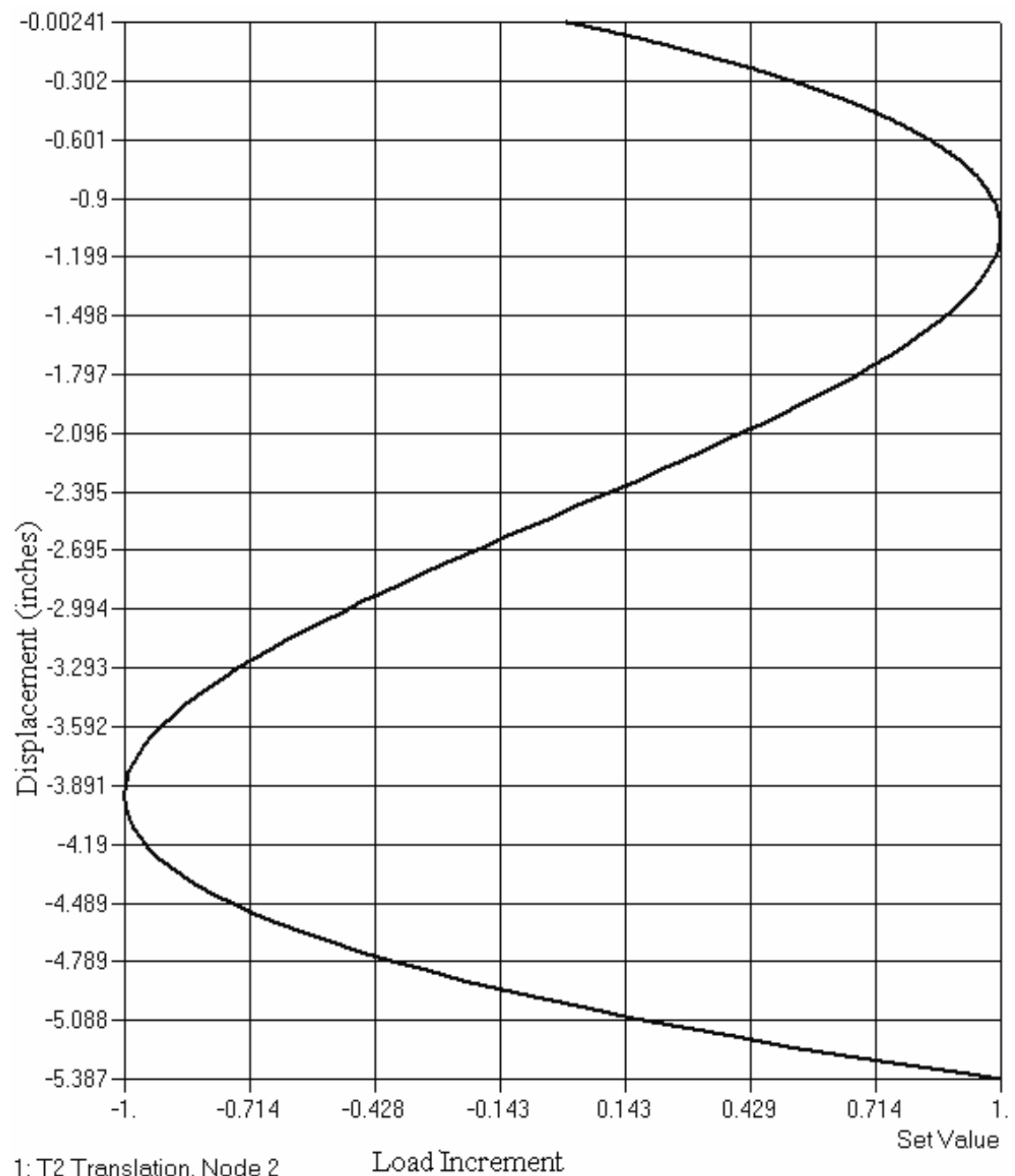

Figure 13. Khot load displacement curve for two member symmetric truss with cross section areas equal to 6.4977 sq. inch.

The published results in [7] used an area of 6.4977 sq. inch and are close to optimal however according to the Nastran results as well as the Hrinda solution they will not support the $200 \mathrm{lb}$. load with snap through buckling still occurring. A Nastran analysis used the smaller area of 6.4977 sq. inch from [7] with the results plotted in figure 13. The equilibrium path shows two critical limit points with the first at a load increment of .99959 or an applied load of $200 \mathrm{lbs}$. times .99959 that equals $199.9180 \mathrm{lbs}$. The first limit point is reached and then the structure responds with a snap through before reaching the intended design load. 


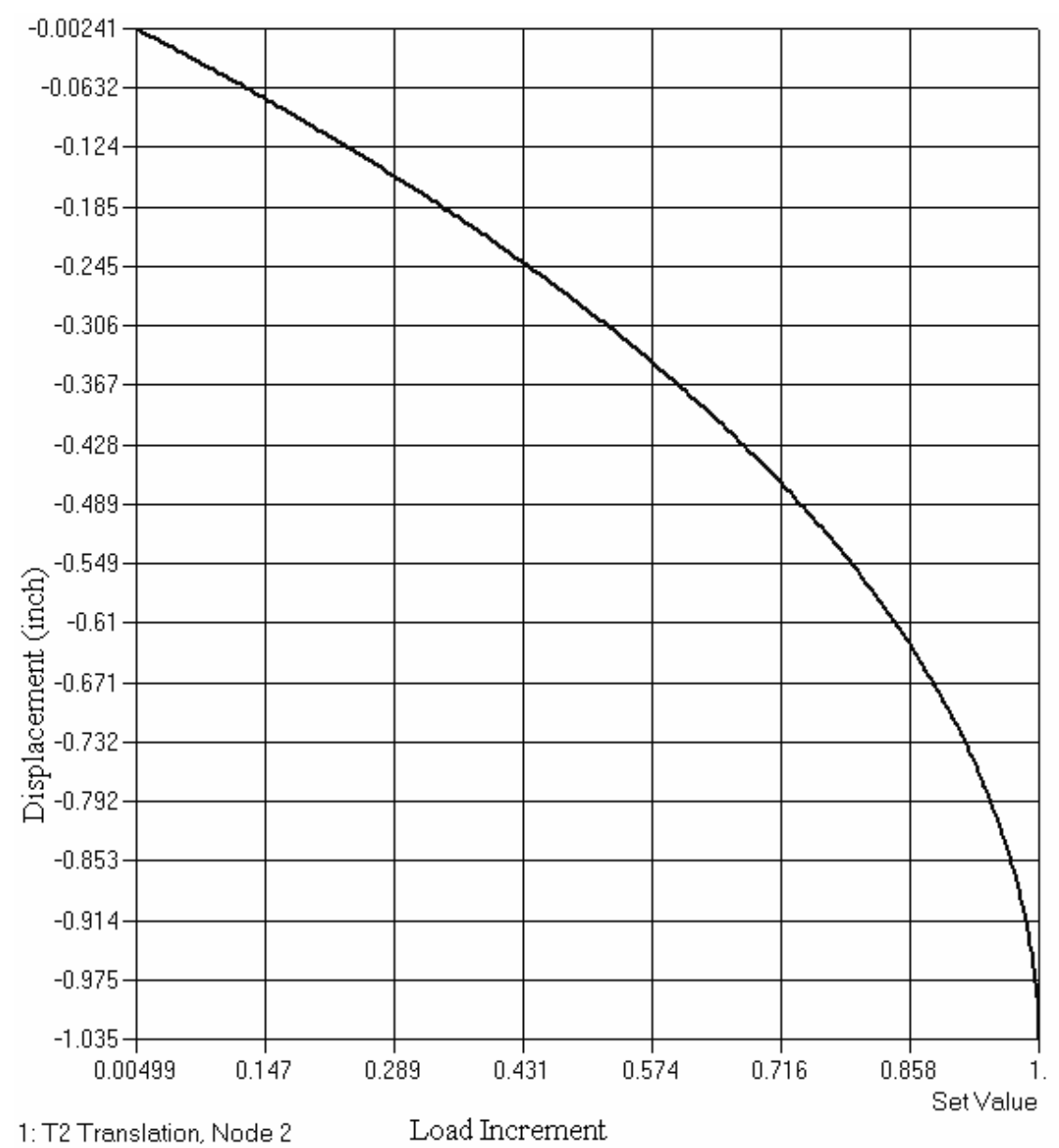

Figure 14. Hrinda load displacement curve for two member symmetric truss with cross section areas equal to 6.4999 sq. inch.

The areas obtained from the Hrinda algorithm were substituted into the nastran model of the two element symmetric truss used in figure 10. The areas for both elements were the same as one expects from symmetry and was 6.4999 sq. inch. This optimal cross section produces a maximum displacement of -1.0354 inch at the maximum load increment of 1.0 as shown in figure 10. The Hrinda obtained area was slightly higher then in [7] and did not cause an instability snap through. The difference between the Hrinda and Khot/Kammat results are practically zero. The two results show the sensitivity of the optimal solution to the number of significant digits used in the design and solution parameters. If a larger load increment were used during the arc length solution then the design area may be slightly smaller as in [7]. The two designs are practically identical but also show how a slight imperfection could affect the design as stated by Bruns[1], Khot and Kammat [7] and Sedaghati [16].

\section{Example 2. Two Member Unsymmetric Truss}

The next verification problem is an unsymmetric two element truss as shown in figure 15 and has the same $200 \mathrm{lb}$. applied load and support conditions as the first example. The problem is also taken from [7]. A summary of the SED results obtained by the Hrinda algorithm is given in figure 16. The optimal area is achieved after the difference between the SED in both 
members fell within the user defined parameter $d S E D t o l$. The final optimal areas were 2.6658 sq. inch and 2.6646 sq. inch or for a practical design they were the same in both members. The weight history shown in figure 17 for each iteration with the final design weight of $66.64 \mathrm{lbs}$. This compared very closely with the results in [7] that where $66.53 \mathrm{lbs}$.

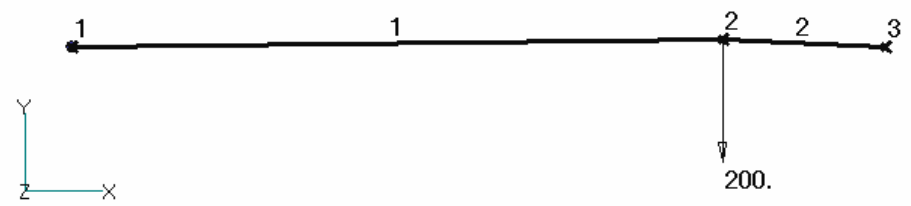

Figure 15. Two element unsymmetric truss.

\begin{tabular}{|c|c|c|c|c|}
\hline $\begin{array}{c}\text { Elemen } \\
\mathrm{t}\end{array}$ & Initial SED & $\begin{array}{c}\text { Initial Relative } \\
\text { SED }\end{array}$ & Final SED & $\begin{array}{c}\text { Final Relative } \\
\text { SED }\end{array}$ \\
\hline 1 & 1.50354 & .24968 & 2.15244 & .99961 \\
\hline 2 & 6.02195 & 1.00000 & 2.15159 & 1.00000 \\
\hline
\end{tabular}

Figure 16. Strain energy density distribution of two element unsymmetric truss.

\begin{tabular}{|c|c|}
\hline Iteration & Weight (lbs.) \\
\hline 1 & $0.4501 \mathrm{E}+02$ \\
\hline 2 & $0.2414 \mathrm{E}+02$ \\
\hline 3 & $0.2461 \mathrm{E}+02$ \\
\hline 4 & $0.2483 \mathrm{E}+02$ \\
\hline 5 & $0.2490 \mathrm{E}+02$ \\
\hline 6 & $0.2492 \mathrm{E}+02$ \\
\hline 7 & $0.2493 \mathrm{E}+02$ \\
\hline 8 & $0.6664 \mathrm{E}+02$ \\
\hline
\end{tabular}

Figure 17. Iteration weight history of two element unsymmetric truss.

\section{Example 3. Four member unsymmetric truss}

The next problem investigated was an optimized unsymmetric four element truss with stability constraints presented by Khot and Kammat [7] and analyzed using the Hrinda optimization approach in this paper. The model is shown in figure 18 with a vertical loading of $200 \mathrm{lbs}$. at the center node. The first part of the analysis was to create a nonlinear Nastran model and test the results in [7] for possible snap through behavior. The areas used in the Nastran analysis were the final design areas in [7] and are shown in figure 20. The load deflection plot of the center node is displayed in figure 22 and shows a critical limit point is reached before the design load of 200. lbs. A critical limit point is reached at a load increment of .9174 with a displacement equal to -1.2597 inches. Strain energy stored in the structure is released and the load decreases with increasing load. The load goes to zero at about -3.00 inches and switches direction until another limit point is reached at a maximum load increment of -.9174 at a displacement equal to -4.7217 inches. The curve given in figure 22 shows how the structure is only able to support a load of .9174 x $200 \mathrm{lbs}$. or $183.48 \mathrm{lbs}$. Figure 19 is a plot of the deflected shape after snap through. 


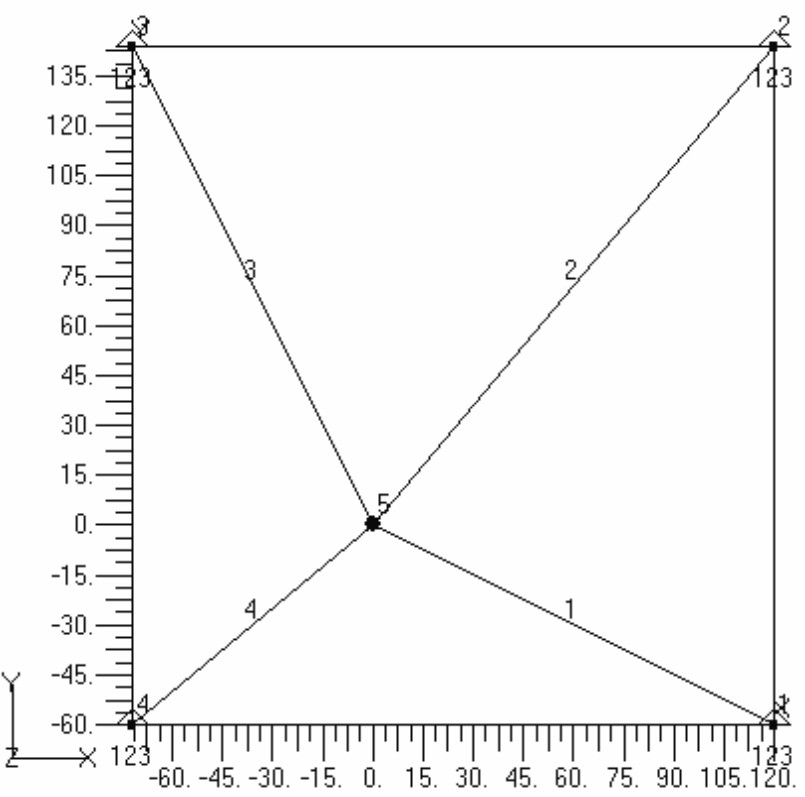

Figure 18. Plan view of the four member unsymmetric truss example.

\begin{tabular}{|c|c|}
\hline Element & Area $\left(\right.$ in $\left.^{2}\right)$ \\
\hline 1 & 2.1555 \\
\hline 2 & 1.6668 \\
\hline 3 & 1.7202 \\
\hline 4 & 2.0894 \\
\hline
\end{tabular}

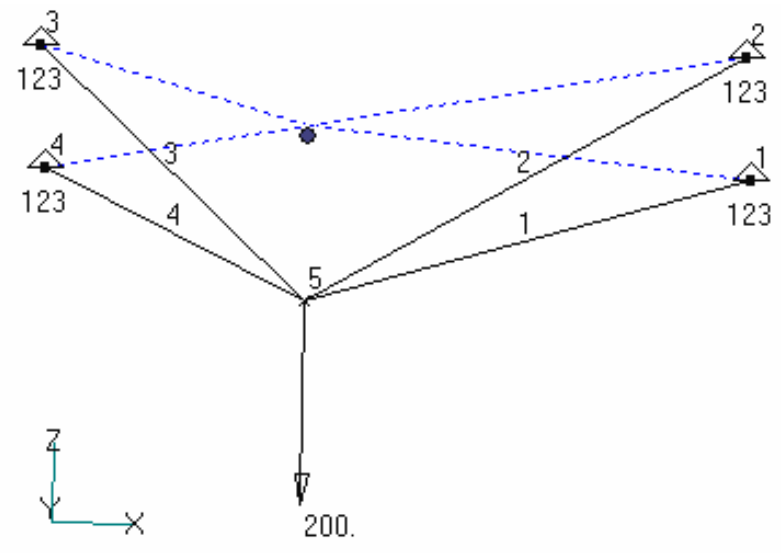

Figure 19. Four member truss at snap through.

Figure 20. Nastran element properties from [7].

Now the proposed Hrinda method is used to solve and optimize the same problem in [7]. The initial areas for all the members were $2.20 \mathrm{sq}$. inch with the final areas shown in figure 21 . This equals an optimized weight of $114.934 \mathrm{lbs}$. as shown in the iteration history in figure 25 . The final Hrinda derived results were used in a Nastran nonlinear analysis with the results for the center node plotted in figure 23.

\begin{tabular}{|c|c|}
\hline Element & Area $\left(\right.$ in $\left.^{2}\right)$ \\
\hline 1 & 2.1613 \\
\hline 2 & 1.6596 \\
\hline 3 & 1.7269 \\
\hline 4 & 2.8940 \\
\hline
\end{tabular}

Figure 21. Hrinda final design. 
The load increment in figure 23 goes to 1.0 with a displacement of -1.248 inches. This plot shows the structure is able to support the $200 \mathrm{lbs}$. load without snap through buckling. The maximum load increment is reached at 1.0 and is multiplied by the applied load of $200 \mathrm{lbs}$. The strain energy density distribution results found by Hrinda are summarized in figure 24 and show that the SED in all members are nearly equal.

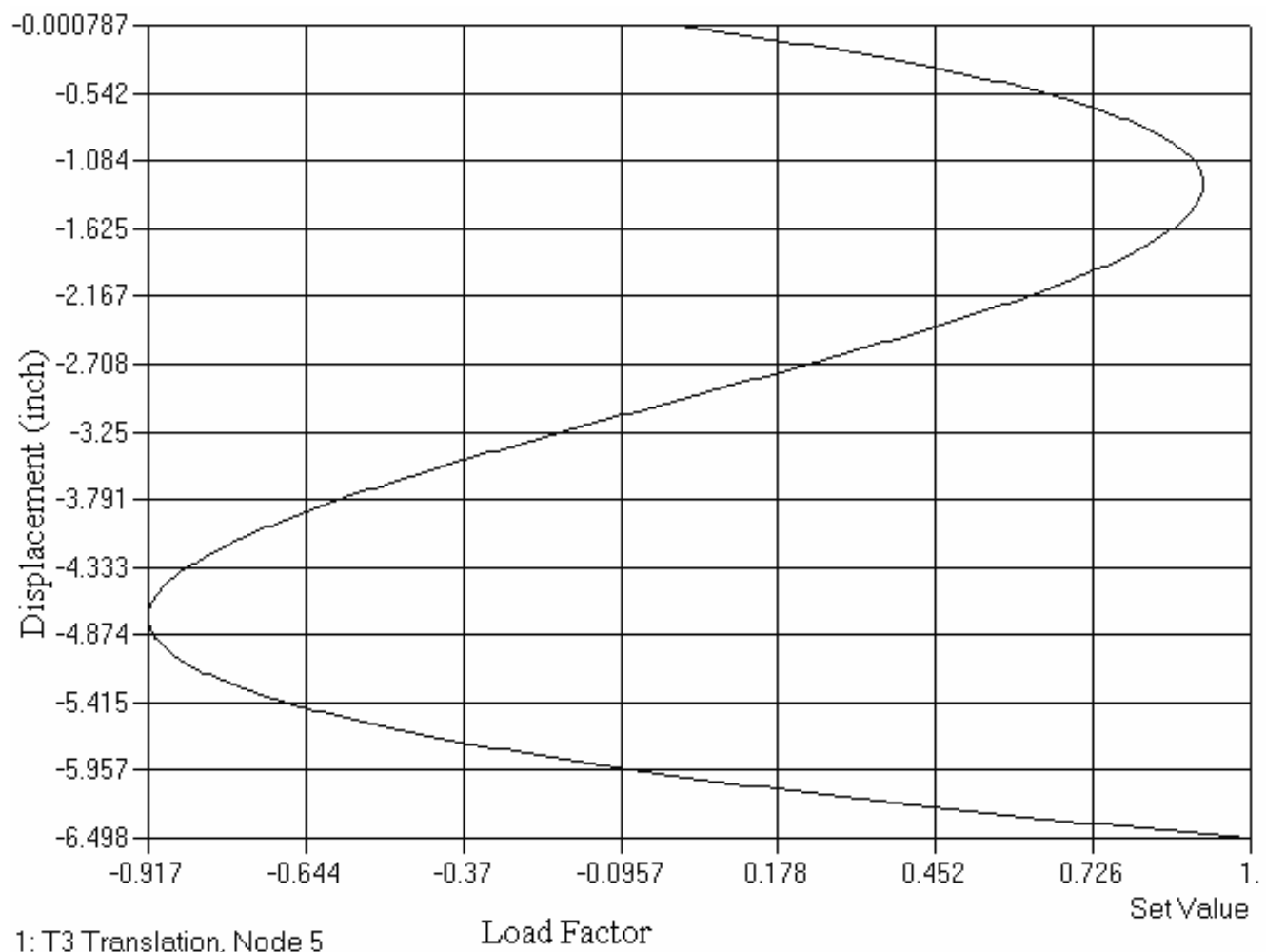

Figure 22. Four member unsymmetric truss equilibrium path during snap through. 


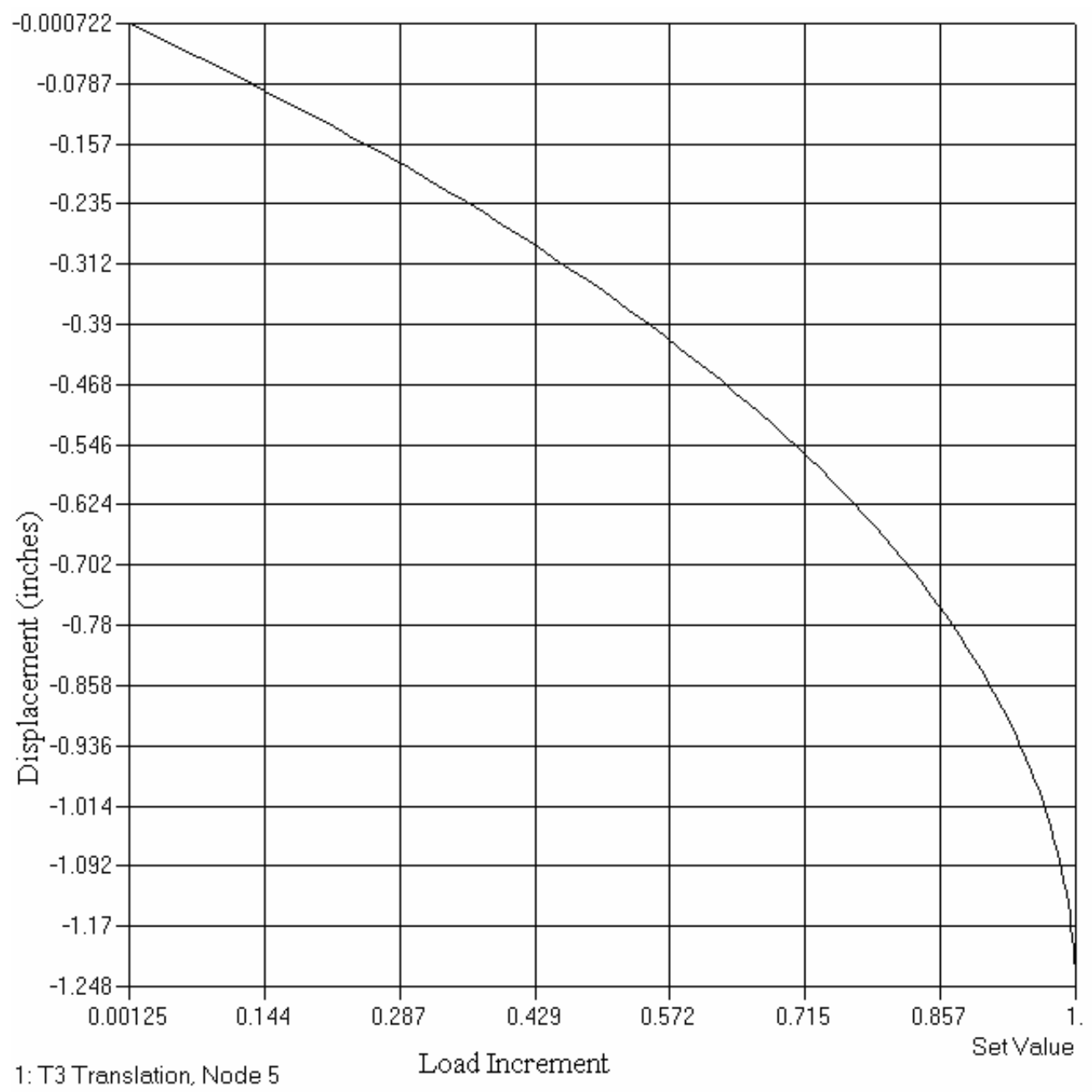

Figure 23. Load displacement results for center node of four member truss using Hrinda results in Nastran.

\begin{tabular}{|c|c|c|c|c|c|c|}
\hline Element & $\begin{array}{c}\text { Initial Area } \\
\left(\text { in }^{2}\right)\end{array}$ & Initial SED & $\begin{array}{c}\text { Initial Relative } \\
\text { SED }\end{array}$ & $\begin{array}{c}\text { Final Area } \\
\left(\text { in }^{2}\right)\end{array}$ & Final SED & $\begin{array}{c}\text { Final Relative } \\
\text { SED }\end{array}$ \\
\hline 1 & 2.20 & $0.105498 \mathrm{E}+01$ & 0.558117 & $0.217240 \mathrm{E}+01$ & $0.150140 \mathrm{E}+01$ & 0.983680 \\
\hline 2 & 2.20 & $0.620698 \mathrm{E}+00$ & 0.328368 & $0.163832 \mathrm{E}+01$ & $0.152631 \mathrm{E}+01$ & 1.000000 \\
\hline 3 & 2.20 & $0.673578 \mathrm{E}+00$ & 0.356343 & $0.172547 \mathrm{E}+01$ & $0.152479 \mathrm{E}+01$ & 0.999004 \\
\hline 4 & 2.20 & $0.189025 \mathrm{E}+01$ & 1.000000 & $0.292330 \mathrm{E}+01$ & $0.147108 \mathrm{E}+01$ & 0.963815 \\
\hline
\end{tabular}

Figure 24. Strain energy density distribution in four member truss.

\begin{tabular}{|c|c|}
\hline Iteration & Weight (lbs.) \\
\hline 1 & $0.126822 \mathrm{E}+03$ \\
\hline 2 & $0.121775 \mathrm{E}+03$ \\
\hline 3 & $0.118580 \mathrm{E}+03$ \\
\hline 4 & $0.116549 \mathrm{E}+03$ \\
\hline 5 & $0.115251 \mathrm{E}+03$ \\
\hline 6 & $0.114934 \mathrm{E}+03$ \\
\hline
\end{tabular}

Figure 25. Iteration weight history of four member truss. 


\section{Example 4. Star Dome Truss}

The next problem presented is the shallow star dome truss example by Khot and Kammat [7] and is shown in figure 26 . The design has thirty members with pin supports at the outer nodes and one vertical load at the center. The design sizing is preformed on four optimization groups that are listed in figure 27. The minimum area for all elements is 0.1 inch sq.

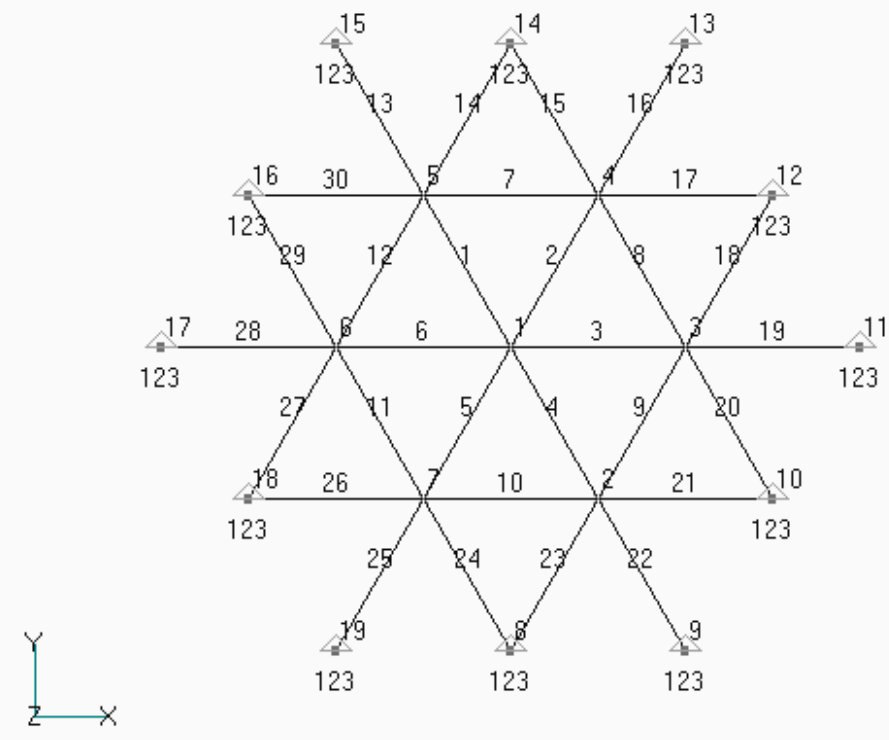

Figure 26. Star dome truss example.

\begin{tabular}{|c|c|}
\hline Group I & $1-6$ \\
\hline Group II & $7-12$ \\
\hline Group III & $13,16,19,22,25,28$ \\
\hline Group IV & $14,15,17,18,20,21$, \\
& $23,24,26,27,29,30$ \\
\hline
\end{tabular}

Figure 27. Star dome optimization groups.

\begin{tabular}{|c|c|c|c|c|c|}
\hline Element & $\begin{array}{c}\text { Initial } \\
\text { SED }\end{array}$ & $\begin{array}{c}\text { Initial } \\
\text { Relative } \\
\text { SED }\end{array}$ & Final SED & $\begin{array}{c}\text { Final } \\
\text { Relative } \\
\text { SED }\end{array}$ & $\begin{array}{c}\text { Final Area } \\
\left(\text { in }^{2}\right)\end{array}$ \\
\hline 1 & 19.428600 & 1.000000 & 15.493200 & 0.964167 & 1.69407 \\
\hline 2 & 19.428600 & 1.000000 & 15.493200 & 0.964167 & 1.69407 \\
\hline 3 & 19.428600 & 1.000000 & 15.493200 & 0.964167 & 1.69407 \\
\hline 4 & 19.428600 & 1.000000 & 15.493200 & 0.964167 & 1.64907 \\
\hline 5 & 19.428600 & 1.000000 & 15.493200 & 0.964167 & 1.64407 \\
\hline 6 & 19.428600 & 1.000000 & 15.493200 & 0.964167 & 1.69407 \\
\hline 7 & 12.605600 & 0.648817 & 15.475700 & 0.963078 & 1.37499 \\
\hline 8 & 12.605600 & 0.648817 & 15.475700 & 0.963078 & 1.37499 \\
\hline 9 & 12.605600 & 0.648817 & 15.475700 & 0.963078 & 1.37499 \\
\hline 10 & 12.605600 & 0.648817 & 15.475700 & 0.963078 & 1.37499 \\
\hline 11 & 12.605600 & 0.648817 & 15.475700 & 0.963078 & 1.37499 \\
\hline 12 & 12.605600 & 0.648817 & 15.475700 & 0.963078 & 1.37499 \\
\hline 13 & 0.740568 & 0.038117 & 16.069000 & 1.000000 & 0.263468 \\
\hline 14 & 0.000002 & 0.000000 & 4.293670 & 0.267202 & 0.100379 \\
\hline 15 & 0.000002 & 0.000000 & 4.293670 & 0.267202 & 0.100379 \\
\hline 16 & 0.740568 & 0.038117 & 16.069000 & 1.000000 & 0.263468 \\
\hline 17 & 0.000002 & 0.000000 & 4.293670 & 0.267202 & 0.100379 \\
\hline 18 & 0.000002 & 0.000000 & 4.293680 & 0.267203 & 0.100379 \\
\hline 19 & 0.740566 & 0.038117 & 16.069000 & 1.000000 & 0.263468 \\
\hline 20 & 0.000002 & 0.000000 & 4.293680 & 0.267203 & 0.100379 \\
\hline 21 & 0.000002 & 0.000000 & 4.293670 & 0.267202 & 0.100379 \\
\hline 22 & 0.740568 & 0.038117 & 16.069000 & 1.000000 & 0.263468 \\
\hline
\end{tabular}




\begin{tabular}{|l|l|l|l|l|l|}
\hline 23 & 0.000002 & 0.000000 & 4.293670 & 0.267202 & 0.100379 \\
\hline 24 & 0.000002 & 0.000000 & 4.293670 & 0.267202 & 0.100379 \\
\hline 25 & 0.740568 & 0.038117 & 16.069000 & 1.000000 & 0.263468 \\
\hline 26 & 0.000002 & 0.000000 & 4.293670 & 0.267202 & 0.100379 \\
\hline 27 & 0.000002 & 0.000000 & 4.293680 & 0.267203 & 0.100379 \\
\hline 28 & 0.740566 & 0.038117 & 16.069000 & 1.000000 & 0.263468 \\
\hline 29 & 0.000002 & 0.000000 & 4.293680 & 0.267203 & 0.100379 \\
\hline 30 & 0.000002 & 0.000000 & 4.293670 & 0.267202 & 0.100379 \\
\hline
\end{tabular}

Figure 28. Star dome strain energy density and truss area design.

\begin{tabular}{|c|c|}
\hline Iteration & Weight(lbs.) \\
\hline 1 & $0.1087 \mathrm{E}+04$ \\
\hline 2 & $0.4963 \mathrm{E}+03$ \\
\hline 3 & $0.5165 \mathrm{E}+03$ \\
\hline 4 & $0.5520 \mathrm{E}+03$ \\
\hline 5 & $0.5913 \mathrm{E}+03$ \\
\hline 6 & $0.6384 \mathrm{E}+03$ \\
\hline 7 & $0.6942 \mathrm{E}+03$ \\
\hline 8 & $0.7622 \mathrm{E}+03$ \\
\hline $\mathbf{9}$ & $\mathbf{0 . 7 6 5 0 E}+\mathbf{0 3}$ \\
\hline
\end{tabular}

Figure 29. Star dome weight summary

\begin{tabular}{|c|c|c|}
\hline Group & $\begin{array}{c}\text { Area }\left(\text { in }^{2}\right) \\
\text { Hrinda }\end{array}$ & $\begin{array}{c}\text { Area }\left(\text { in }^{2}\right) \\
\text { Khot/Kammet }\end{array}$ \\
\hline I & 1.69407 & 1.6926 \\
\hline II & 1.37499 & 1.3754 \\
\hline III & 0.263468 & 0.2693 \\
\hline IV & 0.100379 & 0.1000 \\
\hline
\end{tabular}

Figure 30. Final optimized group truss areas comparison.

The strain energy density summary and final member areas are shown in figure 28. The relative strain energy densities are also given and show group IV being sized to the minimum 0.10 inch sq. Group IV members have been sized to the minimum area so their relative SED will not approach unity. Figure 29 shows the total design weights after each iteration with a final weight of $765.0 \mathrm{lbs}$. This compares well with the final optimized design weight of 766.188 lbs. given by Khot/Kammet [7]. The group sizing results are shown in figure 30 and also show a very close comparison with results from Khot/Kammet.

\section{Example 5. Large Shallow Truss}

The next problem is a large symmetric shallow truss that is presented by Khot and Kammet in [7]. The design is shown in figure 31 and includes 23 members sized for two applied loads of $300 \mathrm{lbs}$. and $600 \mathrm{lbs}$. with symmetric boundary conditions at the apex and pinned at node 1 .

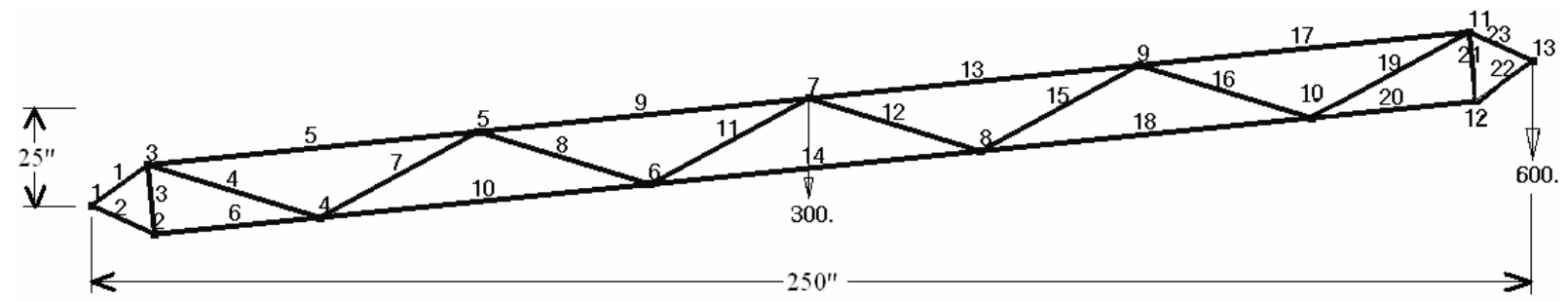

Figure 31 . Shallow truss half symmetry model with two point loads. 


\begin{tabular}{|c|c|c|c|c|c|c|}
\hline Element & $\begin{array}{c}\text { Initial } \\
\text { SED }\end{array}$ & $\begin{array}{c}\text { Initial } \\
\text { Relative } \\
\text { SED }\end{array}$ & $\begin{array}{c}\text { Final } \\
\text { SED }\end{array}$ & $\begin{array}{c}\text { Final } \\
\text { Relative } \\
\text { SED }\end{array}$ & $\begin{array}{c}\text { Final Area } \\
\left(\text { in }^{2}\right) \\
\text { Hrinda }\end{array}$ & $\begin{array}{c}\text { Final Area } \\
\left(\text { in }^{2}\right) \\
\text { Khot }\end{array}$ \\
\hline 1 & 2.65769 & 2.65769 & 1.59397 & 0.99744 & 2.5794 & 2.4747 \\
\hline 2 & 2.30213 & 2.30213 & 1.59178 & 0.99607 & 2.4089 & 2.3695 \\
\hline 3 & 5.75174 & .575174 & 1.56639 & 0.98018 & 1.2110 & 1.1837 \\
\hline 4 & 2.09883 & .020988 & 1.50676 & 0.94287 & 0.2307 & 0.2609 \\
\hline 5 & 2.38905 & 2.38905 & 1.59195 & 0.99618 & 2.4434 & 2.3694 \\
\hline 6 & 1.72627 & 1.72627 & 1.58652 & 0.99278 & 2.0888 & 2.0551 \\
\hline 7 & 2.07244 & .020724 & 1.50816 & 0.94375 & 0.2306 & 0.2259 \\
\hline 8 & 1.99700 & .019970 & 1.50975 & 0.94474 & 0.2292 & 0.2227 \\
\hline 9 & 3.27302 & 3.27302 & 1.59805 & 1.00000 & 2.8546 & 2.7512 \\
\hline 10 & 1.09653 & 1.09653 & 1.57834 & 0.98766 & 1.6750 & 1.6353 \\
\hline 11 & 1.93285 & .019328 & 1.50944 & 0.94455 & 0.2264 & 0.1945 \\
\hline 12 & 1.92360 & .019236 & 1.50927 & 0.94444 & 0.2258 & 0.1941 \\
\hline 13 & 3.23698 & 3.23698 & 1.59784 & 0.99986 & 2.8390 & 2.7463 \\
\hline 14 & 6.21472 & .621472 & 1.56710 & 0.98063 & 1.2654 & 1.2751 \\
\hline 15 & 1.96688 & .019668 & 1.50947 & 0.94457 & 0.2274 & 0.2230 \\
\hline 16 & 2.02073 & .020207 & 1.50774 & 0.94348 & 0.2277 & 0.2249 \\
\hline 17 & 2.36631 & 2.36631 & 1.59177 & 0.99607 & 2.4319 & 2.3649 \\
\hline 18 & 1.09379 & 1.09379 & 1.57828 & 0.98762 & 1.6728 & 1.6351 \\
\hline 19 & 2.06643 & .020664 & 1.50650 & 0.94271 & 0.2289 & 0.2590 \\
\hline 20 & 1.71562 & 1.71562 & 1.58640 & 0.99271 & 2.0824 & 0.0524 \\
\hline 21 & 5.64525 & .564525 & 1.56604 & 0.97996 & 1.1998 & 1.1756 \\
\hline 22 & 2.28075 & 2.28075 & 1.59160 & 0.99596 & 2.3978 & 2.3626 \\
\hline 23 & 2.62850 & 2.62850 & 1.59375 & 0.99730 & 2.5653 & 2.4687 \\
\hline & & & & & & \\
\hline
\end{tabular}

\begin{tabular}{|c|l|}
\hline Iteration & Weight (lbs.) \\
\hline 1 & $0.156524 \mathrm{E}+03$ \\
\hline 2 & $0.134545 \mathrm{E}+03$ \\
\hline 3 & $0.123092 \mathrm{E}+03$ \\
\hline 4 & $0.116857 \mathrm{E}+03$ \\
\hline 5 & $0.113505 \mathrm{E}+03$ \\
\hline 6 & $0.111891 \mathrm{E}+03$ \\
\hline 7 & $0.111382 \mathrm{E}+03$ \\
\hline 8 & $0.111581 \mathrm{E}+03$ \\
\hline 9 & $0.112211 \mathrm{E}+03$ \\
\hline 10 & $0.113076 \mathrm{E}+03$ \\
\hline 11 & $0.114034 \mathrm{E}+03$ \\
\hline 12 & $0.114992 \mathrm{E}+03$ \\
\hline 13 & $0.115892 \mathrm{E}+03$ \\
\hline 14 & $0.116704 \mathrm{E}+03$ \\
\hline 15 & $0.117415 \mathrm{E}+03$ \\
\hline 16 & $0.118024 \mathrm{E}+03$ \\
\hline 17 & $0.118539 \mathrm{E}+03$ \\
\hline 18 & $0.118968 \mathrm{E}+03$ \\
\hline 19 & $0.119322 \mathrm{E}+03$ \\
\hline 20 & $0.119612 \mathrm{E}+03$ \\
\hline $\mathbf{2 1}$ & $\mathbf{0 . 1 1 9 8 4 9 E}+\mathbf{0 3}$ \\
\hline
\end{tabular}

Figure 32. Shallow truss strain energy

Figure 33. Shallow truss weight summary. density and final design areas.

The strain energy density summary and final member areas are shown in figure 32. A $d S E D t o l=0.10$ is imposed for all elements. The Hrinda derived member areas are listed next to Khot [7] results for comparison. All the members are in close agreement except member 20. This is because the Hrinda results imposed a .1 inch sq. minimum area so the optimizer was forced to size accordingly. The Hrinda weight summary is shown in figure 33 with the final weight of $119.85 \mathrm{lbs}$. Doubling this value to account for symmetry, gives the final shallow truss weight of $239.70 \mathrm{lbs}$. This is close to the final weight of $234.13 \mathrm{lbs}$. found in [7].

\section{Conclusions}

The optimization of stability constrained truss structures exhibiting highly nonlinear snap through behavior has been accomplished using the arc length method and the principal of strain energy density. The iterative design variable updating scheme was used in a finite element approach that also used the critical buckling load at snap through as the design load. The problems executed had very close agreement with previous truss examples found in literature and verify the use of the arc length parameters as performance indicators in updating the design variables. The technique was applied to design problems with highly nonlinear trusses prone to global instability failures but has also been tested on linear optimization problems. Initial 
results show that the arc length and SED parameters can be used for finding the lightest truss under linear behavior.

\section{References}

[1] Bruns, T. E., Sigmund, O., and Tortorelli, D. A., Numerical Methods for the Topology Optimization of Structures that Exhibit Snap-Through, Inter. Journal for Numerical Methods in Engineering, 2002, Vol. 55, pp.1215-1237.

[2] Crisfield, M. A., A Fast Incremental/Iterative Solution Procedure that Handles SnapThrough, Comp. \& Struct., Vol. 13, 55-62.

[3] Crisfield, M. A., Non-Linear Finite Element Analysis of Solids and Structures: Volume I Essentials, John Wiley \& Sons 1991.

[4] Crisfield, M. A., Non-Linear Finite Element Analysis of Solids and Structures: Volume 2 Advanced Topics, John Wiley \& Sons 1997.

[5] Greenberg, H. S., Large Space Structures, taken from lecture notes presented June 14-18 at NASA Langley Research Center, 2004.

[6] Kamat, M. P., and Ruangsilasingha, P., Design Sensitivity Derivatives for Structures in Nonlinear Response, AIAA paper no. 84-0973, 1984.

[7] Khot, N. S., and Kamat, M. P., Minimum Weight Design of Truss Structures with Geometric Nonlinear Behavior, AIAA Journal, Vol. 23, No. 1, January 1985.

[8] Khot, N. S., Kamat, M. P., Venkayya, V. B., Optimization of Shallow Trusses Against Limit Point Instability, AIAA Journal, Vol. 22, No. 3, March 1984.

[9] Khot, N.S., Venkayya, V. B. and L. Berke, Optimum structural design with stability constraints, Int. J. Num. Methods Eng. 10 (1976), pp. 1097-1114.

[10] Makris, P. A. and Provatidis, C. G., Weight minimization of displacement-constrained truss structures using a strain energy criterion, Computer methods in applied mechanics and engineering 191, (pp. 2159-2177), 2002.

[11] Makris, P. A., Provatidis, C. G., and Venetsanos, D. T., Structural optimization of thinwalled tubular trusses using a virtual strain energy density approach, Thin-Walled Structures 44, (pp 235-246) Elsevier 2006.

[12] Nguyen, D.T., Finite Element Methods: Parallel-Sparse Statics and Eigen-Solutions, Springer Publisher 2006.

[13] Pai, P. F., Highly Flexible Structures: Modeling, Computation, and Experimentation, AIAA Education Series, 2007. 
[14] Prager, W. Optimality Criteria in Structural Design, Proceedings of the National Academy of Sciences of the United States of America, Vol. 61, No. 3 (Nov. 15, 1968), pp. 794-796.

[15] Prager, W. and Taylor, J.E., Problems of Optimal Structural Design, J. Appl. Mech., Trans. ASME, 90, pp 102-106 (1968).

[16] Sedaghati, R., Tabarrok, B., and Suleman, A., Optimum Design of Nonlinear Symmetric Truss Structure Under System Stability Constraint, 8th AIAA/USAF/NASA/ISSMO Symposium on Multidisciplinary Analysis and Optimization, Sept. 2000, AIAA-2000-4835.

[17] Warren, J. E., Nonlinear Stability Analysis of Frame-Type Structures with Random Geometric Imperfections Using a Total-Lagrangian Finite Element Formulation, $\mathrm{PhD}$. Dissertation, Virginia Polytechnic Institute and State University, January 1997.

[18] West, H. and Geschwindner, L., Fundamentals of Structural Analysis, 2nd edition John Wiley \& Sons 2002.

[19] Xiao-zu, S. and Bashir-Ahmed, M., Arc-length technique for nonlinear finite element analysis, Journal of Zhejiang University Science, pp. 618-628, (2004). 